\title{
A Novel Carbon Price Fluctuation Trend Prediction Method Based on Complex Network and Classification Algorithm
}

\author{
Hua $\mathrm{Xu}^{1,2}$ and Minggang Wang $\mathbb{D}^{1,2,3}$ \\ ${ }^{1}$ School of Mathematical Science, Jiangsu University, Zhenjiang 212013, Jiangsu, China \\ ${ }^{2}$ Taizhou College, Nanjing Normal University, Taizhou 225300, Jiangsu, China \\ ${ }^{3}$ School of Mathematical Science, Nanjing Normal University, Nanjing 210042, Jiangsu, China
}

Correspondence should be addressed to Minggang Wang; magic821204@sina.com

Received 5 October 2021; Accepted 10 December 2021; Published 28 December 2021

Academic Editor: Guilherme Ferraz de Arruda

Copyright (C) $2021 \mathrm{Hua} \mathrm{Xu}$ and Minggang Wang. This is an open access article distributed under the Creative Commons Attribution License, which permits unrestricted use, distribution, and reproduction in any medium, provided the original work is properly cited.

\begin{abstract}
Carbon price fluctuation is affected by both internal market mechanisms and the heterogeneous environment. Moreover, it is a complex dynamic evolution process. This paper focuses on carbon price fluctuation trend prediction. In order to promote the accuracy of the forecasting model, this paper proposes the idea of integrating network topology information into carbon price data; that is, carbon price data are mapped into a complex network through a visibility graph algorithm, and the network topology information is extracted. The extracted network topology structure information is used to reconstruct the data, which are used to train the model parameters, thus improving the prediction accuracy of the model. Five prediction models are selected as the benchmark model, and the price data of the EU and seven pilot carbon markets in China from June 19, 2014, to October 9, 2020, are chosen as the sample for empirical analysis. The research finds that the integration of network topology information can significantly improve the price trend prediction of the five benchmark models for the EU carbon market. However, there are great differences in the accuracy improvement effects of China's seven pilot carbon market price forecasts. Moreover, the forecasting accuracy of the four carbon markets (i.e., Guangdong, Chongqing, Tianjin, and Shenzhen) has improved slightly, but the prediction accuracy of the carbon price trend in Beijing, Shanghai, and Hubei has not improved. We analyze the reasons leading to this result and offer suggestions to improve China's pilot carbon market.
\end{abstract}

\section{Introduction}

At present, global climate change and dioxide emission reduction become hot topics of global concern. Effectively reducing gas emissions and curbing the trend of worldwide warming have become common challenges for all countries within the world. The carbon emission commerce market originates from the theory of environmental property rights and the theory of ecological modernization, which advocate for market means to solve environmental problems. It is a good policy tool to regulate and reduce greenhouse gas emissions by a market mechanism and an important institutional innovation to push green and low-carbon transformation of economic development mode. Since the Kyoto Protocol came into force, the international carbon market has seen rapid growth. By the end of 2018, there have been 20 carbon markets operating around the world, covering $8 \%$ of the world's gas emissions, and the countries and regions where the carbon markets are located account for $37 \%$ of the global economy [1]. As the world's largest emitter and the country with the greatest potential to reduce emissions, China has a path of emission reduction that has drawn close attention from the international community. In its own unique way, it has demonstrated its determination and actions to actively tackle climate change. At the Paris Climate Change Conference 2015, the Chinese government made a solemn commitment to reduce carbon dioxide intensity per unit of GDP by $60-65 \%$ by 2030 from the 2005 level, hit peak carbon emissions around 2030, and raise the share of nonfossil energy in primary energy consumption to 
$20 \%$. In 2020, at the General Debate of the 75th Session of the United Nations General Assembly, President Xi Jinping said that the country should strive to achieve carbon neutrality by 2060, which means that China must reduce its carbon emissions from 16 billion tons per year to almost no emissions during the 40-year period from 2020 to 2060. At present, China is steadily promoting the construction of a national carbon emission trading market. As of November 15,2019 , the cumulative spot trading volume of the carbon market in the pilot areas had reached 364 million tons, and the trading volume had reached 7.985 billion yuan. The total carbon emission and intensity within the pilot range had both reduced, and the low-carbon awareness of enterprises in the pilot market had been continuously improving [2].

The aim of the carbon market is to guide enterprises to create emission-reduction selections through the mechanism of supply and demand to form an effective carbon commerce price. Carbon price fluctuations are influenced not only by internal market mechanisms but also by heterogeneous environments [3]. Carbon price fluctuation is a complex dynamic evolution process. Accurate prediction of carbon price is very important for grasping the fluctuation law of carbon price and avoiding investment risk [4]. Existing carbon price or energy price forecasting models can be roughly divided into three categories. The first is the forecasting model using econometric methods. This kind of model can be further divided into two types. The first is the structural model, such as the error correction model (ECM), vector error correction model (VECM), and vector autoregressive (VAR) model. Such models process data by means of linear regression, which not only includes historical carbon price data but also can include corresponding explanatory variables [3-9]. The second is the time series prediction models, including the autoregressive integrated moving average (ARIMA) model, generalized autoregressive conditional heteroskedasticity (GARCH) model, and their extended models. Such models take no account of other factors and rely only on historical price for forecasts [10-13]. Forecasting models based on econometrics have the advantage of capturing the time-varying fluctuation characteristics of carbon price, but they cannot accurately describe the nonlinear characteristics in the process of carbon price fluctuation. The second category is the prediction model based on machine learning, such as the neural network, random forest, support vector machine, and neurofuzzy control model [14-18]. Compared with the first category model, the second category model has a stronger ability to deal with nonlinear data. However, this kind of model often contains a large number of parameters, and it is prone to defects such as overfitting or poor convergence in the process of use. The third category is a combination prediction model based on multiple methods, such as an integrated model of group method of data handling (GMDH), particle swarm optimization (PSO), and least squares support vector machines (LSSVM), that is, GMDH-PSOLSSVM [19], the integrated model of empirical mode decomposition (EMD), particle swarm optimization (PSO), and support vector machines (SVM), that is, EMD-PSOSVM [20], the hybrid ARIMA and LSSVM methodology
[21], the hybrid approach with exogenous variables [22], the combination of the model based on phase space reconstruction (PSR) and the least squares support vector regression (LSSVR) model [23], the multiscale nonlinear ensemble leaning paradigm [24], the variational mode decomposition and optimal combined model [25], the model based on secondary decomposition algorithm and optimized back propagation neural network [26], the particle swarm optimization (PSO) and radial basis function (RBF) algorithm model [27], the prediction model based on extremum point symmetric mode decomposition, the extreme learning machine and Grey Wolf optimization algorithm [28], and the hybrid method based on empirical wavelet transform (EWT) and Gated Recursive Unit Neural Network (GRU) [29]. This kind of model effectively integrates and utilizes the advantages of the single prediction model, and its prediction accuracy is significantly higher than that of the single prediction model. However, the structure of this kind of model is more complex than the single prediction model, and a large number of model parameters need to be determined, which brings a lot of inconvenience to the practical application. Some typical prediction models are shown in Table 1.

The carbon market has its own unique market characteristics, such as great differences in the price and policy mechanism between the EU and China's pilot carbon markets. The factors affecting the carbon market are numerous, and the structures are complex. Besides, within the background of the current carbon market link, various uncertainties inside and outside the market have become important driving factors affecting the price trend of the carbon market. The price mechanism and fundamental characteristics of the carbon market have changed, and the traditional research framework cannot explain the driving mechanism and characteristics of carbon price fluctuation under uncertain conditions. The existing carbon price prediction models have not fully explored the historical carbon price fluctuation information or the influence of financial high-frequency data, network information, and market uncertainties on carbon price fluctuation. There are still several issues worth discussing within the field of carbon price prediction. Recently, the technology of building complex networks for data has attracted extensive attention from scholars at home and abroad, and a variety of methods to map nonlinear data into complex networks have been proposed [30-33]. In the field of energy economy research, complex network construction technology was used to construct an energy price network based on the energy price data. The fluctuation characteristics of price were studied, and the fluctuation mechanism of price and the dynamic characteristics of the energy price network were revealed $[34,35]$. Based on the data of energy import and export trade, the energy trade network was constructed to reveal the interaction pattern and the evolution characteristics of trade relations between energy import and export countries [36]. Based on the data between the energy market, carbon market, and financial market, the information transmission network among multiple markets was constructed, and the volatility spillover effect of the energy market was studied 
TABLE 1: Summary of studies on price forecasting via various methods.

\begin{tabular}{|c|c|c|c|c|}
\hline Types & Typical literature & Forecasting models & $\begin{array}{l}\text { Data } \\
\text { type }\end{array}$ & Main results \\
\hline \multirow{6}{*}{$\begin{array}{l}\text { Econometric } \\
\text { models }\end{array}$} & Lanza et al. [7] & ECM & Daily & The cointegration marginally improves static forecasts \\
\hline & Murat et al. [8] & VECM & Weekly & VECM outperforms the random walk model (RWM) \\
\hline & $\begin{array}{l}\text { Baumeister et al. } \\
\text { [9] }\end{array}$ & VAR & Monthly & $\begin{array}{l}\text { VAR tends to have lower MSPE at short horizons than the } \\
\text { AR and ARMA models }\end{array}$ \\
\hline & Xiang Y [11] & ARIMA & Daily & $\begin{array}{c}\text { ARIMA model possesses a good prediction effect and can } \\
\text { be used as a short-term prediction }\end{array}$ \\
\hline & Fan et al. [12] & GED-GARCH & Daily & $\begin{array}{l}\text { GED-GARCH model has superior power in the out-of- } \\
\text { sample forecast compared with the popular HSAF method }\end{array}$ \\
\hline & $\begin{array}{l}\text { Mohammadi } \\
\text { et al. [13] }\end{array}$ & $\begin{array}{c}\text { GARCH, EGARCH, } \\
\text { APARCH, and FIGARCH }\end{array}$ & Weekly & APARCH model outperforms other models \\
\hline \multirow{5}{*}{ AI models } & $\begin{array}{l}\text { Atsalakis et al. } \\
{[14]}\end{array}$ & ANN & Daily & ANN model outperforms the ARMA and GARCH models \\
\hline & Yahşi et al. [15] & GEP & Daily & GEP model outperforms the ANN and ARIMA models \\
\hline & Xie et al. [16] & SVM & Monthly & SVM model outperforms the ARIMA and BPNN models \\
\hline & Zhu et al. [17] & LSSVM & Daily & $\begin{array}{l}\text { LSSVM forecasting model outperforms the SVM and RBF } \\
\text { network models }\end{array}$ \\
\hline & Lu et al. [18] & MML & Monthly & $\begin{array}{l}\text { MML model has superior power compared with the } \\
\text { traditional machine learning models }\end{array}$ \\
\hline \multirow{5}{*}{ Hybrid models } & Zhu et al. [19] & GMDH-PSO-LSSVM & Daily & $\begin{array}{l}\text { GMDH-PSO-LSSVM model performs better than the } \\
\text { conventional LSSVM model }\end{array}$ \\
\hline & Gao et al. [20] & EMD-PSO-SVM & Daily & $\begin{array}{l}\text { EMD-PSO-SVM model is significantly superior to the } \\
\text { single SVM model }\end{array}$ \\
\hline & Zhu et al. [21] & $\begin{array}{l}\text { Hybrid ARIMA and } \\
\text { LSSVM methodology }\end{array}$ & Daily & $\begin{array}{l}\text { ARIMA-LSSVM models outperform their single } \\
\text { benchmarks in both level and directional predictions }\end{array}$ \\
\hline & Huang et al. [27] & PSO-RBF & Monthly & $\begin{array}{l}\text { PSO-RBF approach is able to improve prediction accuracy } \\
\text { and to simplify the complexity of the RBF model structure }\end{array}$ \\
\hline & Liu et al. [29] & EWT-GRU & Daily & $\begin{array}{l}\text { The proposed approach is significantly effective and } \\
\text { practically feasible }\end{array}$ \\
\hline
\end{tabular}

[37]. The above research used the network science to study the energy economic system and to obtain many valuable results. The rise and development of complex network science give an alternative perspective and methodology for energy economic data mining and carbon price trend prediction.

In fact, higher prediction accuracy depends on the suitability of the prediction model and the quality of the data used to train the model. Therefore, reconstructing the collected data and using the reconstructed data to train the prediction model are effective means to improve the prediction accuracy. Therefore, this paper puts forward the idea of integrating network topology information into carbon price data. Compared with previous research works, the contribution and innovation of this paper lie in the following aspects: (1) With respect to the research objects, previous studies mostly focused on the real value of carbon price, while this paper focuses on the fluctuation trend, because in many cases, such as policy-making and scenario analysis, it is enough to know only the information about the rise and fall trend of carbon price. Therefore, it is of great practical significance to predict the rise and fall trend of carbon price. (2) From the perspective of improving the prediction algorithm of carbon price fluctuation trend, a new prediction paradigm is proposed based on complex network and the classical discriminant analysis algorithm. This paper proposes the idea of integrating network topology information into carbon price data. The classical discriminant analysis algorithm is trained with the data set, and then the carbon price trend prediction model is constructed. The degree of improvement of the prediction accuracy of the benchmark model after incorporating the topological structure information of the carbon price network is quantitatively analyzed, which was not found in previous studies. (3) In terms of sample data selection, previous studies mostly analyzed the price data of a specific carbon market, but this paper selects the price data of eight carbon markets for comparative analysis and discusses the effect of different carbon market price data on the prediction accuracy.

The structure of the rest of this paper is as follows. The second part details the methods used in this paper. The third part describes how we build the carbon price visibility graph network and analyze the network structure. In the fourth part, we make a comparative analysis of the predicted results. In the fifth part, we provide the conclusion.

\section{Methodology}

2.1. Visibility Graph Algorithm. A visibility graph is a method to map time series into a complex network. As proposed by Lacasa et al., the basic idea is to treat each data point of time series as a network node and to set up an edge between nodes if the "visual condition" is satisfied. The specific mathematical description is as follows: Let $\left\{x\left(t_{i}\right)\right\}_{i=1,2, \ldots N}$ be a time series containing $N$ data, and there is 
an edge between node $i$ and node $j$ if and only if the following visual criteria are met:

$$
x\left(t_{k}\right)<x\left(t_{i}\right)+\left[x\left(t_{j}\right)-x\left(t_{i}\right)\right] \frac{t_{k}-t_{i}}{t_{j}-t_{k}}, \quad t_{i}<t_{k}<t_{j} .
$$

According to the visibility graph algorithm, a time series containing $N$ data can be mapped into a network containing $N$ nodes. The principle of a visibility graph is simple, and the visibility graph network obtained has connectivity properties such as undirected affine invariance and limited information loss. It can also effectively distinguish a random sequence from a chaotic sequence. The complexity of the visibility graph algorithm is $O\left(n^{2}\right)$; thus, it needs a lot of computation time in practical application, which restricts its application in practice. To reduce the time complexity of the algorithm, Luque et al. (2010) modified the visual criterion as follows [38]:

$$
x\left(t_{i}\right), x\left(t_{j}\right)>x\left(t_{k}\right), \quad \text { for any } t_{i}<t_{k}<t_{j} .
$$

This algorithm is called the horizontal visibility graph algorithm (HVG). This algorithm not only maintains the related properties of the visibility graph algorithm but also carries out theoretical analysis on specific time series. The complexity of the algorithm is $O(n)$, which greatly reduces the computational complexity compared with the visibility graph algorithm and has a higher application value.

2.2. Network Topology Index. Many concepts and methods have been proposed to characterize the statistical characteristics of complex network structures, such as degree distribution, average path length, and clustering coefficient. In the following, we only introduce the topological indicators used in the reconstruction of data.

2.2.1. Clustering Coefficient. The clustering coefficient is a coefficient that measures the degree of the cluster of nodes in the network [39]. The local clustering coefficient of node $i$ is expressed as

$$
C_{i}=\frac{2\left|e_{i}\right|}{k_{i}\left(k_{i}-1\right)}
$$

where $\left|e_{i}\right|$ represents the number of connecting edges between nodes in the neighborhood of node $i, k_{i}$ is the degree of node $i$, and $C_{i} \in[0,1]$.

2.2.2. Network Assortativity Coefficient. In the nonzero graph $G=(V, E), u_{e}$ and $v_{e}$ represent the degree of two nodes on some edge $e \in E$, and $N$ is the number of edges in graph $G$. Then, the assortativity coefficient $r$ of the network is

$r=\frac{\left(N^{-1}\right) \sum_{e \in E}\left(u_{e}+v_{e}\right)-\left[\left(N^{-1} / 2\right) \sum_{e \in E}\left(u_{e}+v_{e}\right)\right]^{2}}{\left(N^{-1} / 2\right) \sum_{e \in E}\left(u_{e}^{2}+v_{e}^{2}\right)-\left[\left(N^{-1} / 2\right) \sum_{e \in E}\left(u_{e}+v_{e}\right)\right]^{2}}$,

where the assortativity coefficient is a degree-based Pearson correlation coefficient. When $r$ is positive, nodes with a high degree of connection tend to other nodes with a high degree of connection. When $r$ is negative, nodes with a high degree tend to nodes with a low degree of connection [40].

2.3. Classification Algorithm. Classification is an important research area in data mining, machine learning, and pattern recognition. By analyzing the data of training samples of known categories, one can find classification rules and predict categories of new data. The classification algorithms involved in this paper include linear discriminant analysis (LDA), naive Bayes (NB), K-nearest neighbor (KNN), random forest (RF), and support vector machine (SVM).

2.3.1. $L D A$. LDA is a dimension-reduction technology with supervised learning. Its idea is to project data that are not easy to classify in a certain direction so that intraclass variance can be minimized and interclass variance can be maximized after the projection. That is, after the projection, the projection points of each kind of data are expected to be as close as possible, while the distance between the data category centers of different categories is as large as possible [41].

Let $N$ be the number of data samples, $L$ be the number of classes, $N_{i}$ be the number of class $i$ samples, and $x_{j}^{(i)}$ be the $j$ sample of class $i$. Then, the linear discriminant analysis can be expressed by the following optimization function:

$$
\mathbf{V}=\underset{V}{\arg \max } \frac{\mathbf{V}^{T} \mathbf{S}_{b}^{\mathrm{LDA}} \mathbf{V}}{\mathbf{V}^{T} \mathbf{S}_{w}^{\mathrm{LDA}} \mathbf{V}}
$$

where $\mathrm{V}$ is the projection matrix, $\mathbf{S}_{b}^{\mathrm{LDA}}$ is the divergence matrix between classes, and $\mathbf{S}_{w}^{\mathrm{LDA}}$ is the divergence matrix within the following class:

$$
\begin{aligned}
& \mathbf{S}_{b}^{\mathrm{LDA}}=\sum_{i=1}^{L-1} \sum_{j=i+1}^{L} \frac{N_{i}}{N} \frac{N_{j}}{N}\left(m_{i}-m_{j}\right)\left(m_{i}-m_{j}\right)^{T}, \\
& \mathbf{S}_{w}^{\mathrm{LDA}}=\sum_{i=1}^{L} \sum_{j=1}^{N_{i}} \frac{1}{N}\left(x_{j}^{(i)}-m_{i}\right)\left(x_{j}^{(i)}-m_{i}\right)^{T},
\end{aligned}
$$

where $m_{i}=1 / N_{i} \sum_{j=1}^{N_{i}} x_{j}^{(i)}$.

2.3.2. Naive Bayes (NB) Model. Although LDA is simple and intuitive, it does not consider that the observation value of each category is different and the opportunity of each category is different, nor does it take into account the difference of losses caused by misjudgment. Naive Bayes discrimination can overcome the above shortcomings, and its calculation process is as follows. The possible estimate of each sample that may belong to a certain population (category) is called the "prior probability," denoted as $P\left(G_{i}\right)$. The value of the prior probability can be obtained from experience or can be estimated using the percentage of each group of samples in the total sample [42]. The score of each sample can be calculated according to the discriminant function, and the conditional probability of the discriminant score $S$ under the condition that it belongs to the = category is $P\left(S / G_{i}\right)$. The probability of the sample being judged as a certain $G_{i}$ 
category according to the discriminant function is called the postprobability. According to the Bayesian formula, the posterior probability can be calculated as

$$
P\left(\frac{G_{i}}{s}\right)=\frac{P\left(S / G_{i}\right) P\left(G_{i}\right)}{\sum P\left(S / G_{i}\right) P\left(G_{i}\right)} .
$$

Each sample is classified according to the posterior probability of being assigned to a category.

In summary, the basic idea of Bayesian discrimination is as follows. For each sample, the score of the discriminant function is first calculated. Then, according to the prior probability, $P\left(G_{i}\right)$, and the conditional probability, $P\left(S / G_{i}\right)$, of the discriminant score, $S$, the posterior probability $P\left(G_{i} / S\right)$ of each category is calculated for the sample to be judged. The category in which the posterior probability is the largest is judged, and the sample is judged as the category.

2.3.3. K-Nearest Neighbor (KNN). The basic idea of $K N N$ is as follows. If most of the $k$-nearest neighbors or most similar samples of a sample belong to a category, then the sample also belongs to that category. In this method, the nearest neighbor samples selected by a sample are all samples that have been correctly classified. This method of discrimination or classification is one of the simplest methods in data mining classification technology [43].

$\mathrm{RF}$ is an advanced algorithm of the decision tree algorithm. Its advantage is that it does not produce overfitting. Its theoretical basis is the law of large numbers, which is simply described as follows. Let $X$ be the sample vector, $Y$ be the label vector of correct classification, and RF consist of a series of single plant classifiers $\left\{h\left(X, \theta_{k}\right) ; k=1,2, \ldots\right\}$, where $\left\{\theta_{k}\right\}$ is an independent identically distributed random variable. Then, the marginal function is defined as

$$
m_{g}(X, Y)=a v_{k} I\left[h_{k}(x)=y\right]-\max _{j \neq y} a v_{k} I\left[h_{k}(x)=j\right] \text {, }
$$

where $I(\cdot)$ is the indicator function and $a v(\cdot)$ is the average value function. The larger the marginal function, the higher the confidence of classification.

2.3.4. Support Vector Machine (SVM). SVM is a linear classifier with the largest interval in the feature space. Its learning strategy is to maximize the interval, and it is ultimately transformed into a convex quadratic programming problem to solve [44]. Based on the existing training set,

$$
T=\left\{\left(x_{1}, y_{1}\right),\left(x_{2}, y_{2}\right), \ldots,\left(x_{l}, y_{l}\right)\right\} \in(X \times Y)^{l},
$$

where $x_{i} \in X=R^{n}, X$ is the input space, each point $x_{i}$ in the input space is composed of $n$ attribute features, and $y_{i} \in Y=\{-1,1\}, i=1,2, \ldots, l$.

Find $R^{n}$ real valued function $g(x)$ on $R^{n}$, so that the classification function $f(x)=\operatorname{sgn}[g(x)]$ can be used to infer the corresponding value of $y$ for any pattern $x$. The mathematical structure expression of vector machine optimization problem is as follows:

$$
\begin{aligned}
& \min \Phi(w)=\frac{1}{2} w^{T} w \\
& \text { s.t. } y_{i}\left(w^{T} x_{i}+b\right) \geq 1, \forall i=1,2, \ldots, \ell
\end{aligned}
$$

The local duality theorem is needed to solve the optimization model shown in (10). The solution process is as follows. First, (10) is expressed as follows:

$$
\begin{aligned}
& \min L(w, b, \alpha)=\frac{1}{2} w^{T} w-\sum_{i=1}^{\ell} \alpha_{i}\left[y_{i}\left(w^{T} x_{i}+b\right)-1\right], \\
& \text { s.t. } \alpha_{i} \geq 0, \quad \forall i=1,2, \ldots, \ell .
\end{aligned}
$$

If $\phi$ is the feature mapping, (10) can also be expressed as $\min L(w, b, \alpha)=\frac{1}{2} w^{T} w-\sum_{i=1}^{\ell} \alpha_{i}\left[y_{i}\left(w^{T} \phi\left(x_{i}\right)+b\right)-1\right]$,

s.t. $\alpha_{i} \geq 0, \quad \forall i=1,2, \ldots, \ell$.

If soft separation is considered and the relaxation variable $\xi$ is introduced, the mathematical structure of the (10) support vector machine optimization problem can be modified as

$$
\begin{aligned}
& \min \Phi(w, \xi)=\frac{1}{2} w^{T} w+C \sum_{i=1}^{\ell} \xi_{i}, \quad C>0, \\
& \text { s.t. } \xi_{i} \geq 0, \quad y_{i}\left(w^{T} x_{i}+b\right) \geq 1-\xi_{i}, \forall i=1,2, \ldots \ell .
\end{aligned}
$$

2.4. Prediction Algorithm Integrating Network Topology Information. The carbon price trend prediction model integrating network topology information proposed in this paper includes three modules:

Step 1. Construct data sets. Let $X=\{x(t)\}$ be carbon price series; then the volatility series $P=\{p(t)\}$ can be calculated by

$$
p(t)=\frac{x(t)-x(t-1)}{x(t-1)},
$$

where $x(0)=x(1), p(1)=0$. The following data matrix $P_{d}$ can be got from the volatility series,

$$
P_{d}=\left[\begin{array}{cccc}
p(1) & p(2) & \ldots & p(L) \\
p(\ell+1) & p(\ell+2) & \ldots & p(\ell+L) \\
\vdots & \vdots & \vdots & \vdots \\
p(n \ell+1) & p(n \ell+2) & \ldots & p(n \ell+L)
\end{array}\right],
$$

where $L$ is the length of the sliding window, $\ell$ is the step, and $n$ is the number of the sliding windows. The independent variable data set $P_{d}^{X}$ is 


$$
\begin{gathered}
P_{d}^{X}=\left[\begin{array}{cccc}
p(1) & p(2) & \ldots & p(L-1) \\
p(\ell+1) & p(\ell+2) & \ldots & p(\ell+L-1) \\
\vdots & \vdots & \vdots & \vdots \\
p(n \ell+1) & p(n \ell+2) & \ldots & p(n \ell+L-1)
\end{array}\right], \text { and the } \\
\text { dependent variable data set } P_{d}^{Y} \text { is } \\
P_{d}^{Y}=[p(L), p(\ell+L), \ldots, p(n \ell+L)] \text {. }
\end{gathered}
$$

Step 2. Construct the visibility graph network and extract carbon price volatility information. We transform each row of the independent variable data set into a complex network using a visibility graph algorithm and get $n$ visibility graph networks, denoted as $\delta=[\delta(1), \delta(2), \ldots, \delta(n)]^{\prime}$. The mean clustering coefficient

$$
C=[C(1), C(2), \ldots, C(n)]^{\prime},
$$

and the network assortativity coefficient

$$
r=[r(1), r(2), \ldots, r(n)]^{\prime},
$$

can be obtained by using ( 3 and 4 ). Then, the new independent variable data set is

$$
\mathrm{NP}_{d}^{X}=\left[\begin{array}{ccccc}
p(1) & \ldots & p(L-1) & C(1) & r(1) \\
p(\ell+1) & \ldots & p(\ell+L-1) & C(2) & r(2) \\
\vdots & \vdots & \vdots & \vdots & \vdots \\
p(n \ell+1) & \ldots & p(n \ell+L-1) & C(n) & r(n)
\end{array}\right] .
$$

Step 3. Divide training data and test data for prediction. The training data and test data are divided into independent variable data sets $\mathrm{NP}_{d}^{X}$, and corresponding label sets are obtained from data set $P_{d}^{Y}$.

The algorithm flowchart is shown in Figure 1.

2.5. Precision Index. The carbon price trend prediction problem can be transformed into a binary classification problem, and based on the prediction result, we can get the following confusion matrix:

\begin{tabular}{lcc}
\hline $\begin{array}{l}\text { Predictive valueReal } \\
\text { value }\end{array}$ & Positive & Negative \\
\hline $\begin{array}{l}\text { Positive } \\
\text { Negative }\end{array}$ & TP (true positive) & FN (false negative) \\
\hline
\end{tabular}

2.5.1. Accuracy. The percentage of the total number of correctly predicted samples in the total number of samples, abbreviated as $\mathrm{AC}$, is calculated by the following formula:

$$
\mathrm{AC}=\frac{\mathrm{TP}+\mathrm{TN}}{\mathrm{TP}+\mathrm{FN}+\mathrm{FP}+\mathrm{TN}} \text {. }
$$

2.5.2. Precision. This index is an evaluation index for the prediction results. Among the results predicted by the model as positive samples, the percentage of the truly positive

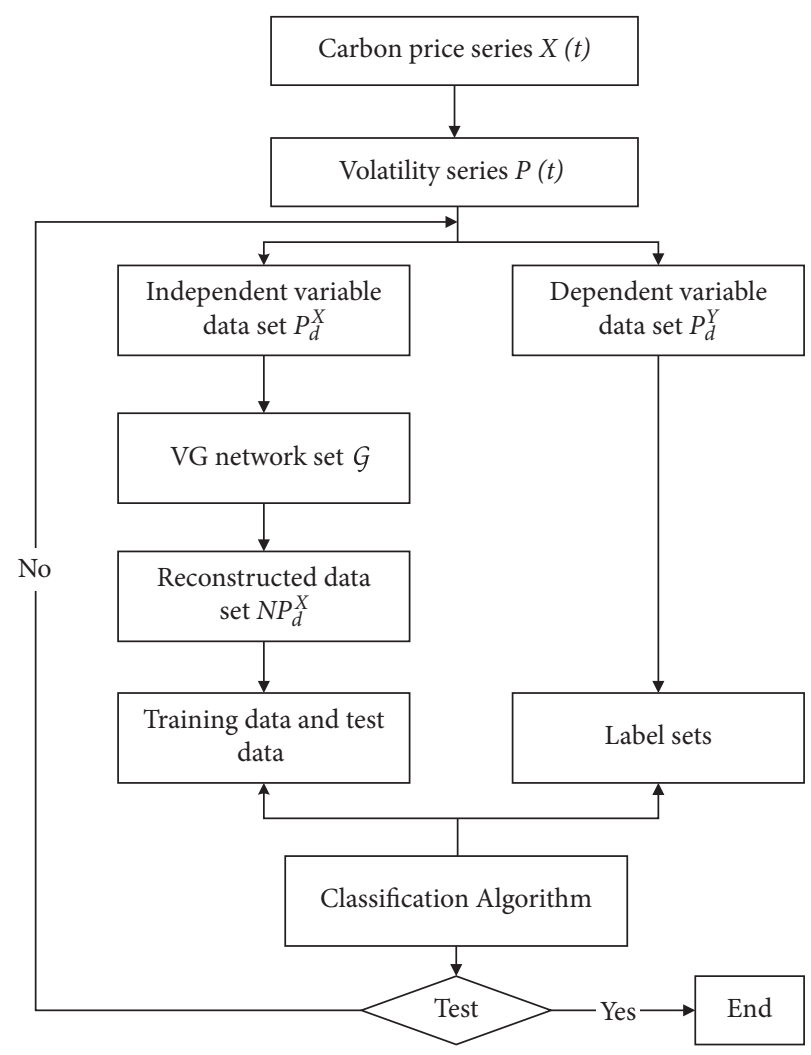

FIgURE 1: Flowchart of the prediction algorithm with network topology information integration.

samples, abbreviated as $\mathrm{PR}$, is calculated by the following formula:

$$
\mathrm{PR}=\frac{\mathrm{TP}}{\mathrm{TP}+\mathrm{FP}}
$$

2.5.3. Recall. This index is an evaluation index for the original sample. Among the actual positive samples, the percentage that is predicted to be positive samples is abbreviated as RE. The calculation formula is as follows:

$$
\mathrm{RE}=\frac{\mathrm{TP}}{\mathrm{TP}+\mathrm{FN}} .
$$

2.5.4. F1 Score. This index is the harmonic mean of PR and $\mathrm{RE}$, taking into account the advantages of both. The calculation formula is as follows:

$$
F 1=\frac{2 \times \mathrm{PR} \times \mathrm{RE}}{\mathrm{PR}+\mathrm{RE}} .
$$

\section{Data Selection and Network Feature Analysis}

The carbon price data of the EU carbon market (EUETS) and seven pilot carbon markets of China from June 19, 2014, to October 9, 2020, that is, Beijing (BJ), Guangdong (GD), Chongqing (CQ), Shanghai (SH), Tianjin (TJ), Shenzhen (SZ), and Hubei (HB), are selected as sample data. For the 

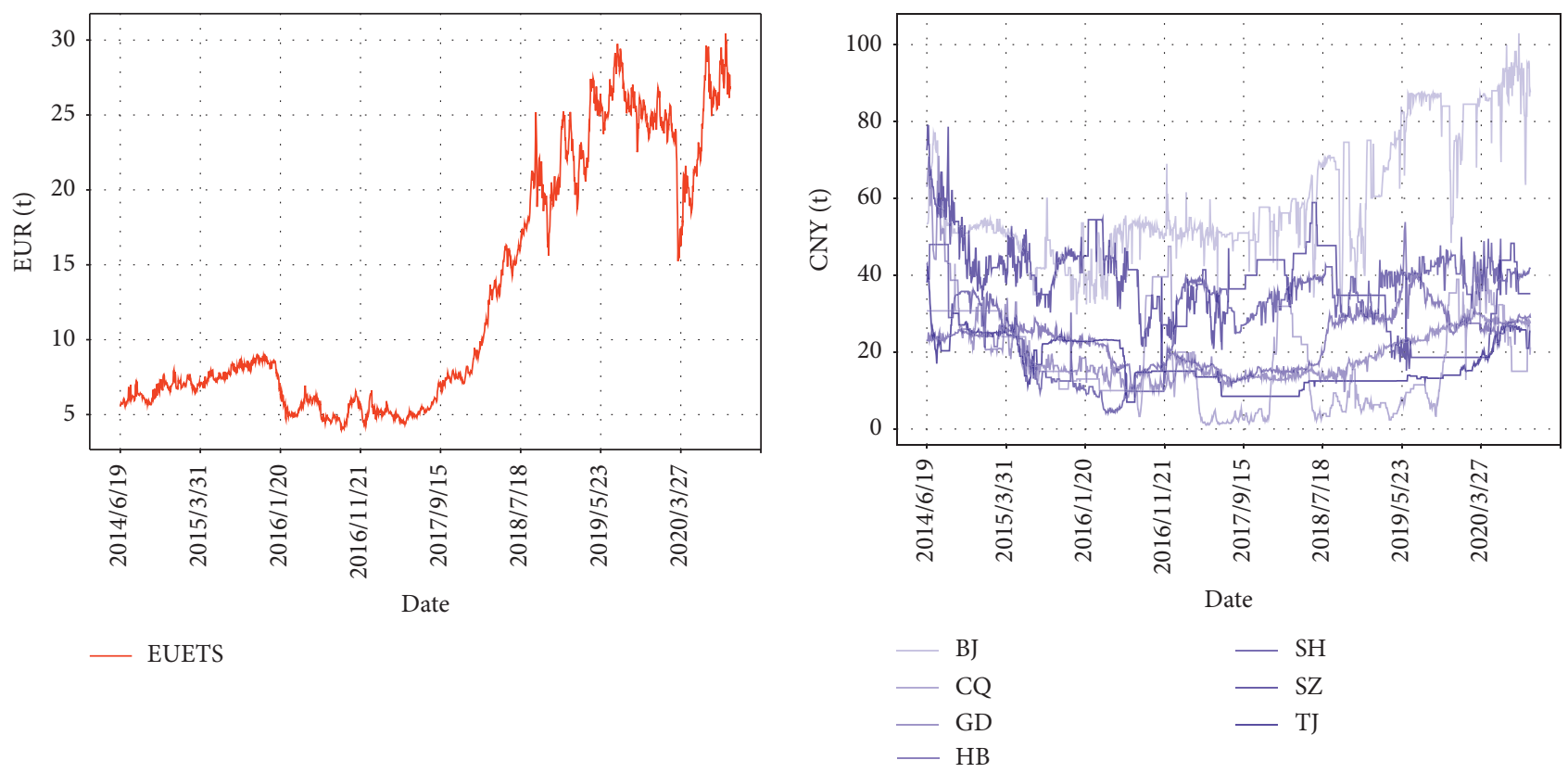

(a)

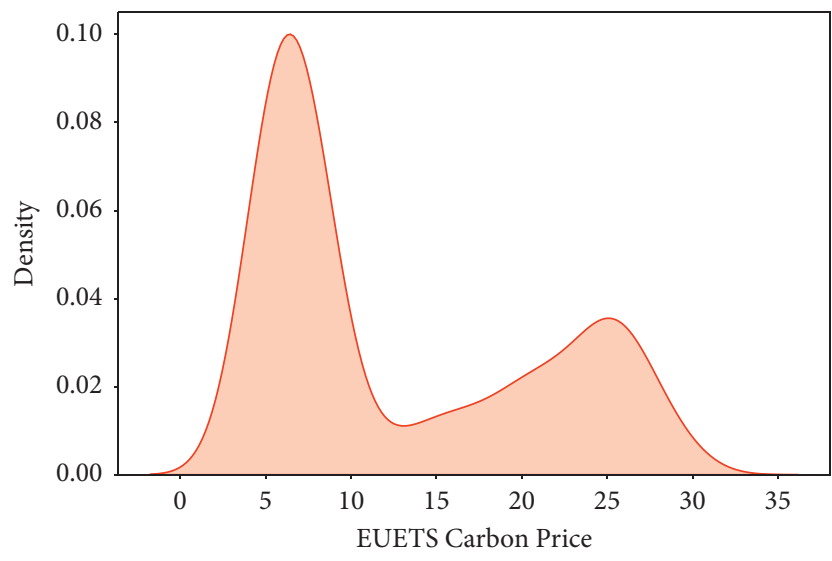

(c)

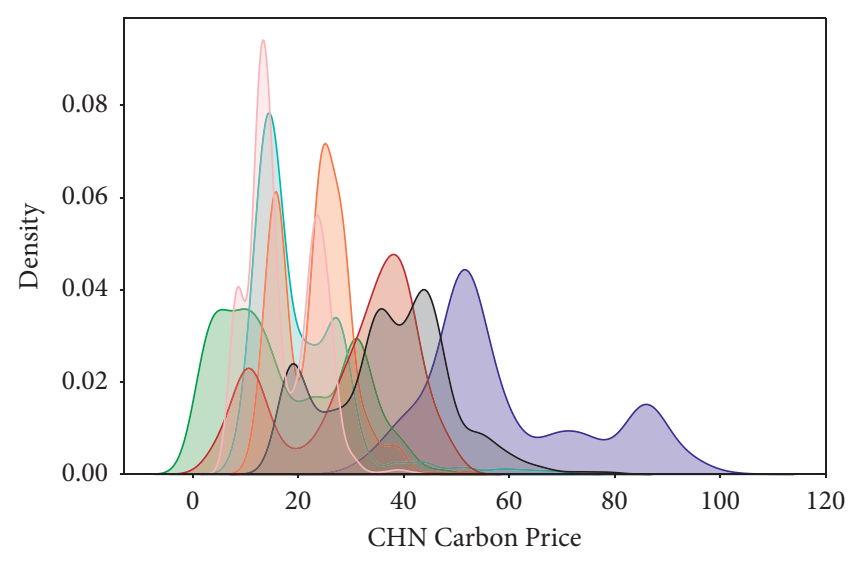

(d)

FIgURE 2: Sample data of carbon prices and their descriptive statistics.

convenience of comparative analysis, we select the time points from June 19, 2014, to October 9, 2020, when data are available in all the eight carbon markets. Therefore, 1525 data samples are selected for each carbon market. The selected sample data of carbon price are shown in Figure 2(a), the statistical image of the sample is shown in Figure 2(b), the kernel density distribution image of the sample is shown in Figure 2(c), and the statistical relationship diagram among the eight sample data of carbon market price is shown in Figure 2(d). The calculation results of descriptive statistics of selected sample data are shown in Table 2 (upper part).

As Figures 2(a) and 2(b) show, the carbon price in China's pilot carbon market is low. The average carbon price in the EU carbon market is $12.8107 \mathrm{EUR} / \mathrm{t}$, and the highest carbon price is $30.44 \mathrm{EUR} / \mathrm{t}$. However, for China's pilot carbon market, Beijing's carbon market has the highest average carbon price, at $59.5397 \mathrm{CNY} / \mathrm{t}$, while TJ carbon market has the lowest average carbon price, at only 16.8758 $\mathrm{CNY} / \mathrm{t}$. According to the coefficient of variation, shown in Table 2, the coefficient of variation of the EU carbon market is greater than the average of China's pilot carbon market, indicating that the fluctuation range of the carbon price in the EU carbon market is higher than the average fluctuation range of China's pilot carbon markets. According to the measurement of the distribution symmetry of the data in Figures 2(c) and 2(d), the EU carbon price data present a right-skewed distribution, indicating that, for the EU carbon market price, the distribution of the carbon price above the mean is more dispersed. Except for Shanghai, China's six pilot carbon markets present a right-skewed distribution. From the perspective of flat data distribution, the EU, Beijing, Chongqing, Shanghai, Shenzhen, and Tianjin carbon markets show flat distribution, while the Guangdong and Hubei carbon market price data show a sharp distribution. As the correlation calculated, there is a significant 
TAвLE 2: Statistical characteristics and network topological characteristics.

\begin{tabular}{|c|c|c|c|c|c|c|c|c|c|}
\hline & & EUETS & $\mathrm{BJ}$ & CQ & GD & HB & $\mathrm{SH}$ & SZ & $\mathrm{TJ}$ \\
\hline \multirow{3}{*}{ Statistical characteristics } & Variable coefficient & 5.3513 & 4.2544 & 7.8148 & 4.2452 & 1.8888 & 4.9197 & 3.6052 & 2.3324 \\
\hline & Skewness & 0.6565 & 0.7156 & 0.3907 & 2.1528 & 0.4177 & -0.7117 & 0.1120 & 0.5065 \\
\hline & Peakness & -1.2343 & -0.6000 & -1.0946 & 6.5115 & 0.3696 & -0.7700 & -0.0632 & -0.4468 \\
\hline \multirow{4}{*}{ Network characteristics } & Average degree & 15.7154 & 10.6964 & 19.6289 & 15.0807 & 16.2544 & 13.4059 & 12.1862 & 26.6584 \\
\hline & Average clustering coefficient & 0.7012 & 0.7688 & 0.7274 & 0.7422 & 0.7262 & 0.7585 & 0.8001 & 0.6848 \\
\hline & Assortativity coefficient & 0.0850 & -0.0652 & -0.3381 & -0.0628 & -0.0823 & -0.0879 & -0.2943 & -0.3984 \\
\hline & Power law index & 1.3959 & 1.5577 & 1.1736 & 1.1354 & 1.1450 & 1.3849 & 1.1820 & 0.9711 \\
\hline
\end{tabular}

positive correlation between the carbon price in the EU carbon market and that in Beijing, Hubei, and Shanghai, with Pearson correlation coefficients of $0.8025,0.7175$, and 0.5535 , respectively. Moreover, there is a negative correlation between the carbon price in the EU carbon market and that in Shenzhen, with a correlation coefficient of -0.4862 .

The eight carbon market price data samples are mapped into a complex network using the visibility graph algorithm, and the corresponding network structure and degree distribution are obtained, as shown in Figure 3. The corresponding network topology index calculation results are shown in Table 2 (the bottom part).

As shown in Figure 3 and Table 2, from the perspective of network topology, the price networks of the EU carbon market and the seven China's pilot carbon markets both have a large degree and high clustering coefficient, and the node degree distribution follows power law distribution. However, the power index is different. The power law index of the EU carbon market is obviously higher than the average value of China's carbon market, indicating that the heterogeneity of the EU carbon market price network is higher than that of China's carbon market price network. From the perspective of network assortativity, the EU carbon market price network assortativity coefficient is greater than 0 , indicating that the network is in assortative, while China's seven carbon markets are on the contrary, indicating that the price network is an assortativity network. Thus, the EU carbon market has some similarities with China's seven pilot carbon markets in terms of statistical characteristics and network topology, but there are significant differences in specific indicators such as the assortativity coefficient. These differences are mainly caused by differences in policies, systems, distribution patterns, and ranges of industries covered by each carbon market.

\section{Model Construction and the Results Analysis}

4.1. Data Reconstruction. We first group the carbon price data of each carbon market at $N=1525$ time points. We set the length of the sliding window as $L$ and the sliding step size as 1 . Then, the price data of $1 \times N$ dimension of each carbon market are transformed into the price data of $(N-L+1) \times L$ dimension, the price data of the first $L-1$ day of each carbon market are taken as the independent variable data set $X$, and the price of the $L t h$ day is taken as the dependent variable $Y$. In this case, the price of the Lth day can be predicted by using the data of the previous $L-1$ days. To integrate network topology information into independent variables, we use the visibility graph algorithm to convert the price data of the first $L-1$ days of each carbon market into a complex network, calculate the average clustering coefficient and assortativity coefficient of each network, and add the calculated results to the independent variable data set $X$. Then, the dimension of the newly constructed data set $X$ is $(N-L+1) \times(L+1)$, and we use this data set to predict the price trend of the carbon market. To test the robustness of the model, we randomly divide the reconstructed data set into a training set and test set. The sample ratio of the training set and test set is set at 9: 1. To illustrate the overall prediction effect of the model, we make several predictions on the test set to calculate the average value of the AC, PR, RE, and F1 score. The numerical simulation software used in this work is Python.

4.2. Analysis of the Forecast Results of the EU Carbon Market. According to the carbon price data of the EU ETS, we use the model to predict the fluctuation trend. We randomly divide the training set and the test set, train the model parameters on the training set, make predictions on the test set by using the trained model, and repeat calculations of $500,1000, \ldots$, 20,000 times. The evolution image of the prediction accuracy index (AC and F1), along with the calculation times, is obtained, as shown in Figure 4.

As Figures 4(a)-4(e) show, the prediction accuracy of the five benchmark prediction models, LDA, KNN, NB, RF, and SVM, and the corresponding CN-LDA, CN-KNN, CN-NB, $\mathrm{CN}-\mathrm{RF}$, and CN-SVM, all tend to be stable with the increase in iteration times. The prediction models incorporating the network topological information are superior to the corresponding benchmark models in terms of prediction accuracy, precision, recall, and F1 value.

Next, we calculate the average prediction accuracy of various models under different iterations, and the results are shown in Table 3.

We analyze the accuracy and F1 value of the model prediction. From Figure 4(f) and Table 3, we find that the average prediction accuracies of the five benchmark prediction models are $0.53822,0.49710,0.53325,0.52835$, and 0.52342 , and the variation coefficients are 0.00092, 0.00118, $0.00087,0.00092$, and 0.00073 . Moreover, the average prediction accuracies of CN-LDA, CN-KNN, CN-NB, CN-RF, and CN-SVM are 0.54253, 0.52877, 0.53618, 0.52952, and 0.52905 , respectively, and the coefficients of variation were $0.00089,0.00102,0.00068,0.00089$, and 0.00072 , respectively. The average predicted F1 values of the five benchmark prediction models are $0.60250,0.53035,0.55835,0.58243$, 


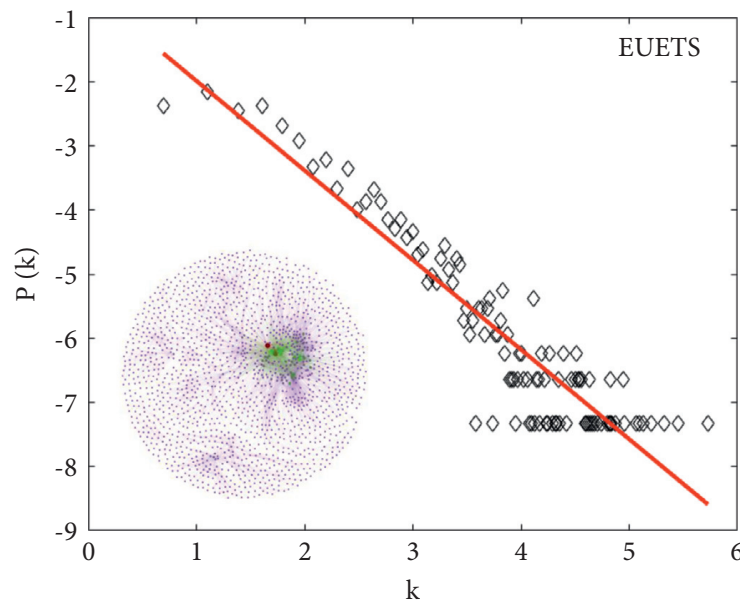

(a)

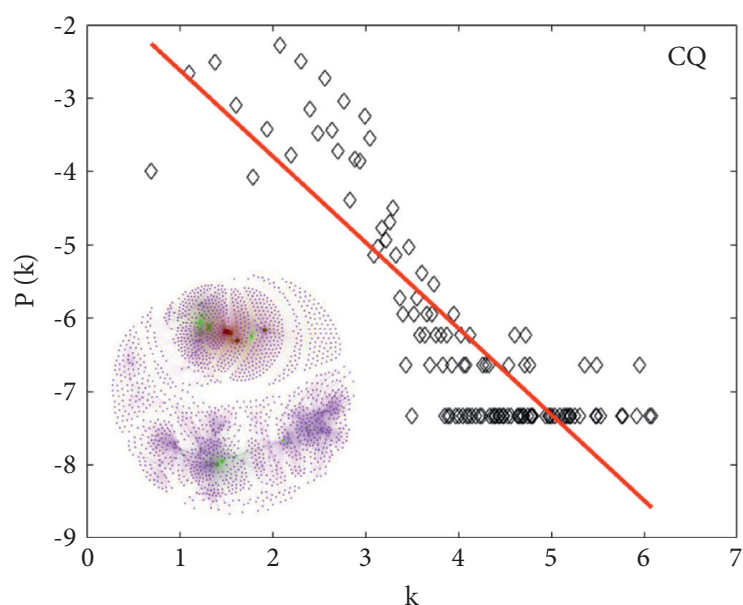

(c)

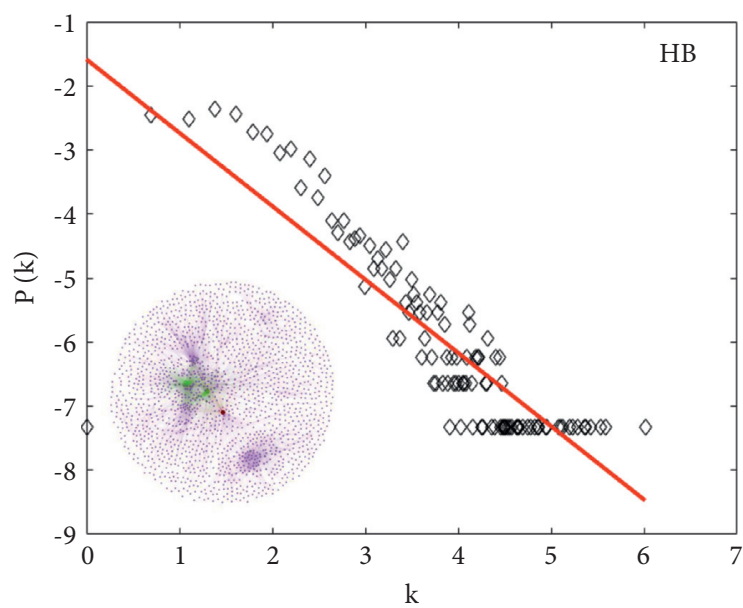

(e)

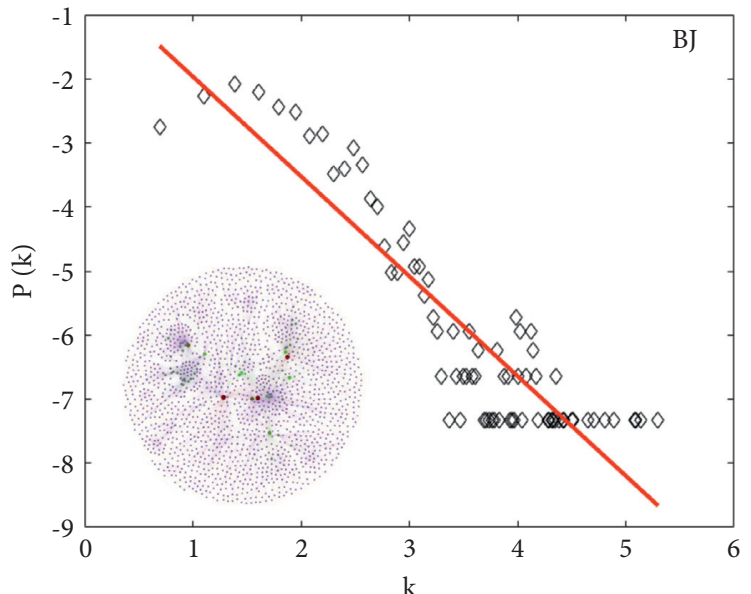

(b)

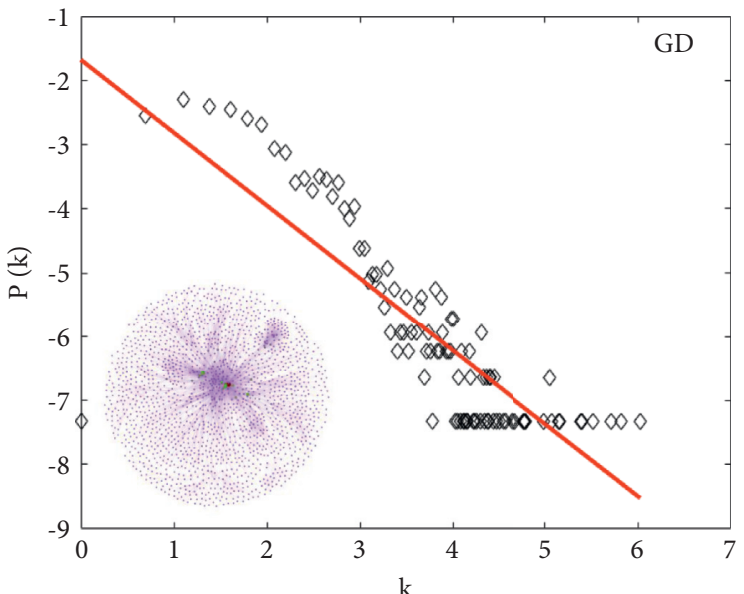

(d)

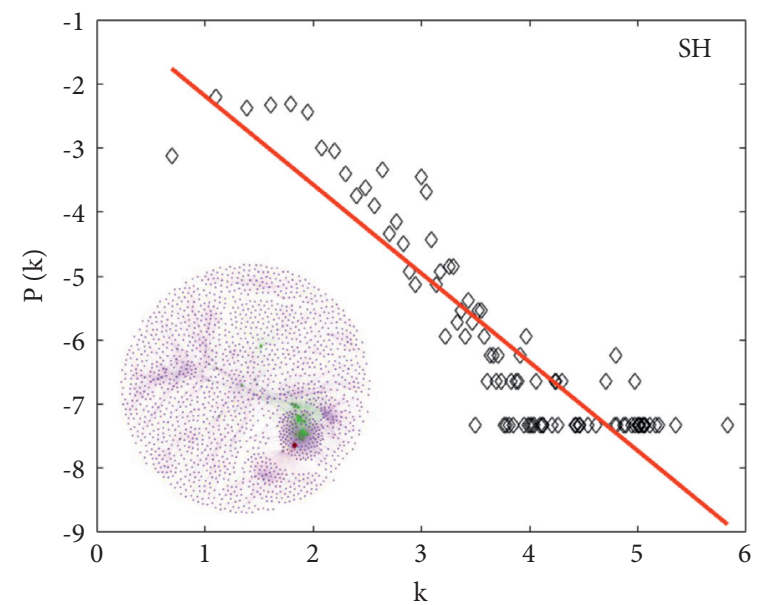

(f)

Figure 3: Continued. 


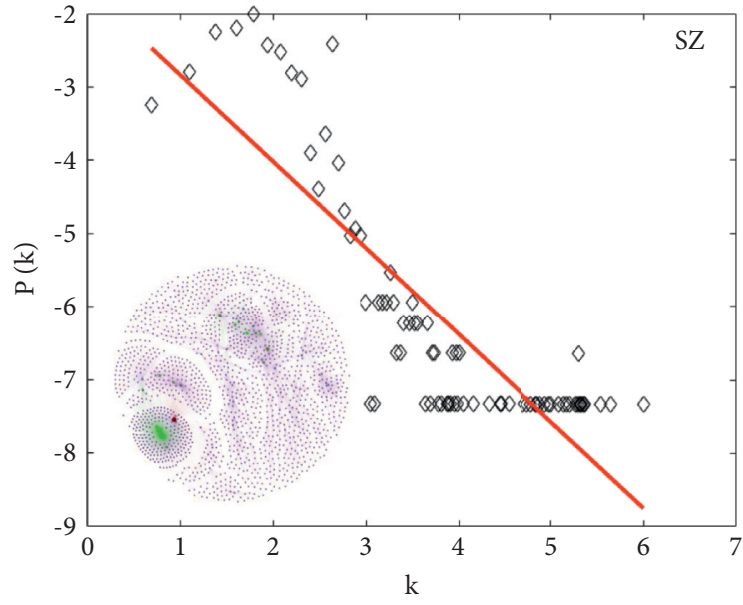

(g)

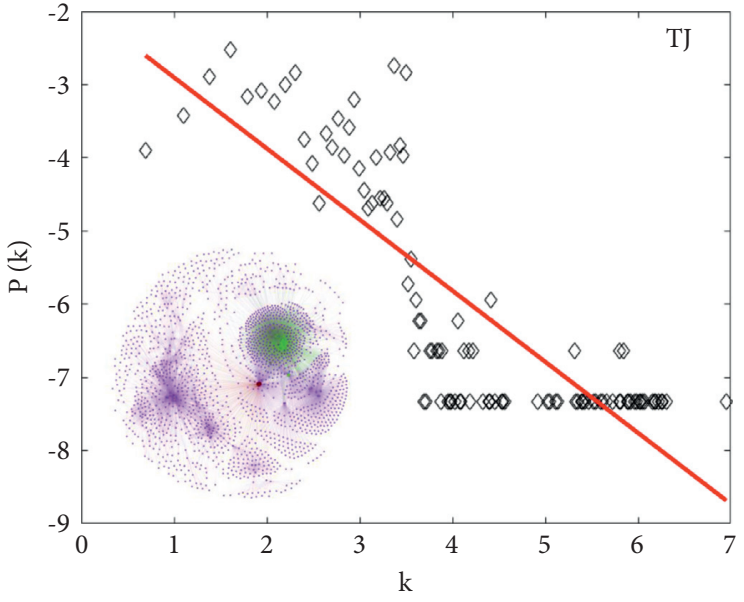

(h)

FIGURE 3: Sample data of carbon prices and their descriptive statistics.
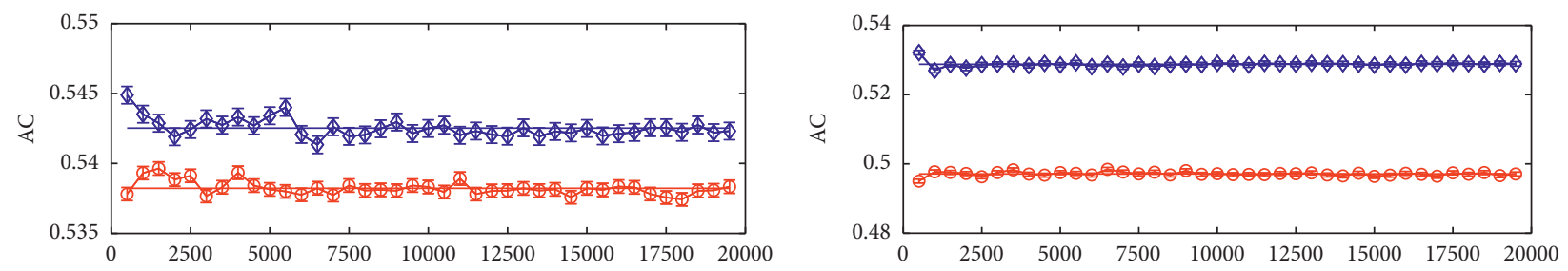

$\multimap$ LDA

$\diamond$ CN-LDA

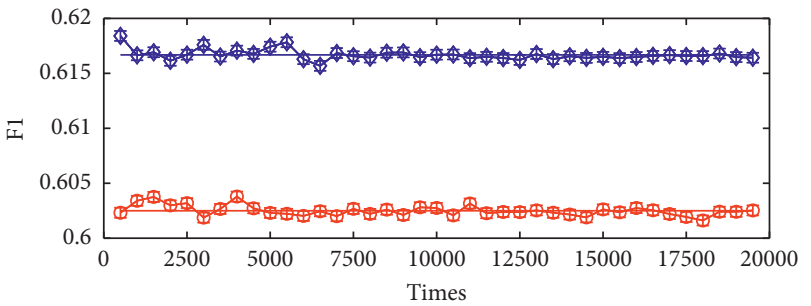

$-\mathrm{KNN}$

$\diamond \mathrm{CN}-\mathrm{KNN}$

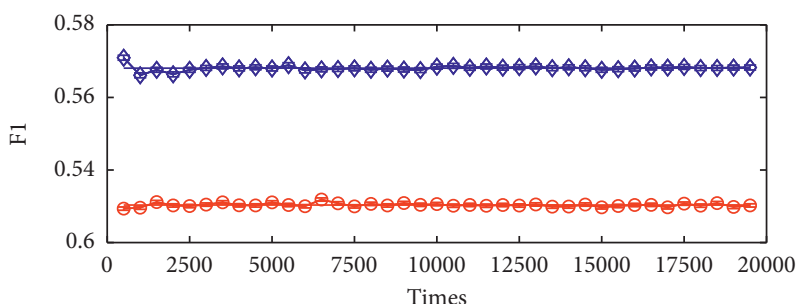

$\multimap$ LDA

$\neg$ CN-LDA

$\multimap \mathrm{KNN}$

$\multimap \mathrm{CN}-\mathrm{KNN}$

(a)

(b)

Figure 4: Continued. 

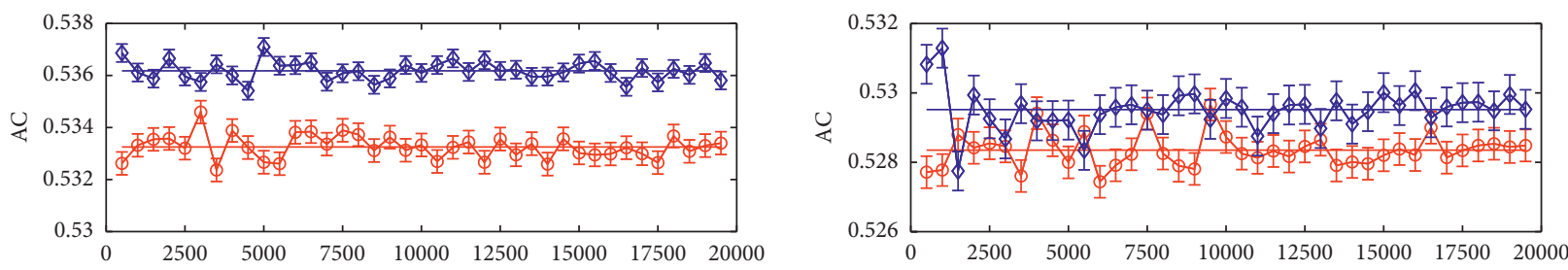

$\circ \mathrm{NB}$

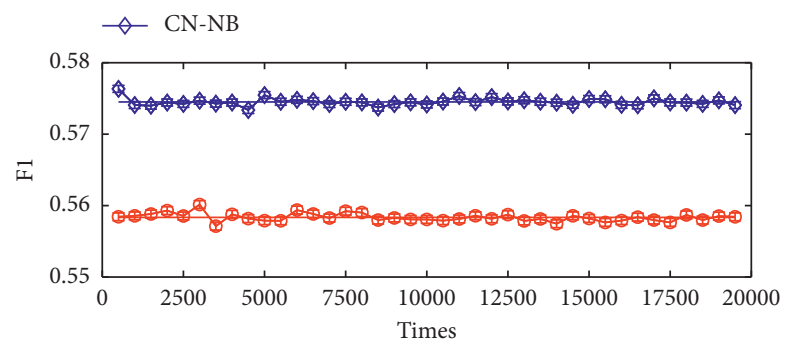

$\multimap \mathrm{RF}$

$\diamond \mathrm{CN}-\mathrm{RF}$

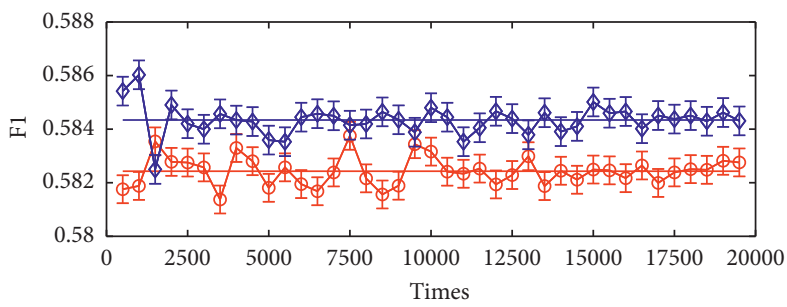

$\multimap$ NB

$\neg$ CN-NB

$-\mathrm{RF}$

$\neg \mathrm{CN}-\mathrm{RF}$

(c)

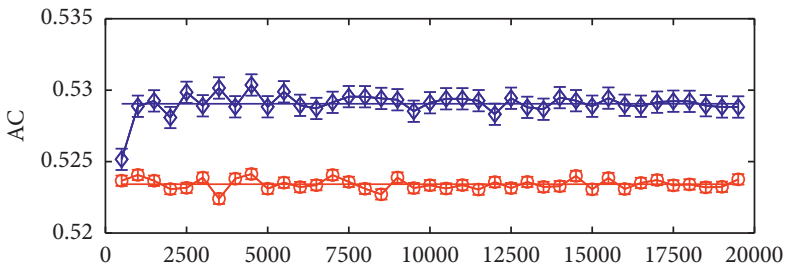

- SVM

$\neg$ CN-SVM

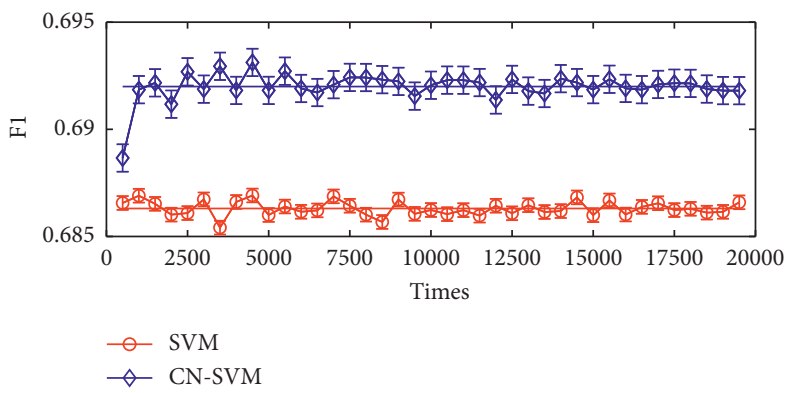

(e)

(f)

Figure 4: Comparison of the predicted results of different forecasting models on the fluctuation trend of carbon price in the EU carbon market.

and 0.68631 , and the variation coefficients are 0.00080 , $0.00091,0.00104,0.00094$, and 0.00049 . Furthermore, the average predicted $\mathrm{F} 1$ values of the corresponding $\mathrm{CN}$ LDA, CN-KNN, CN-NB, CN-RF, and CN-SVM prediction models are $0.61668,0.56807,0.57451,0.58434$, and 0.69200 , respectively. The coefficients of variation are $0.00077,0.00088,0.00089,0.00092$, and 0.00046 . Thus, the prediction accuracy of the CN-LDA model is improved by $0.79952 \%$, and the F1 value is improved by $2.35392 \%$ compared with the LDA model. Compared with the KNN model, the prediction accuracy of the CN$\mathrm{KNN}$ model is increased by $6.37085 \%$, and the $\mathrm{F} 1$ value is increased by $7.11328 \%$. Compared with the NB model, the prediction accuracy of the CN-NB model is increased by $0.54876 \%$, and the $\mathrm{F} 1$ value is increased by $2.89425 \%$. The prediction accuracy of the CN-RF model is $0.22106 \%$ higher than that of the RF model, and the F1 value is $0.32818 \%$ higher than that of the RF model. Moreover, compared with the SVM model, the prediction accuracy of the CN-SVM model is increased by $1.07535 \%$, and the $\mathrm{F} 1$ value is increased by $0.82907 \%$. Furthermore, from the results of the coefficient of variation calculation, it can be seen that the stability of the prediction results of the $\mathrm{CN}$ LDA, CN-KNN, CN-NB, CN-RF, and CN-SVM prediction models is better than that of the corresponding benchmark models. 
TABLE 3: The results of the average prediction accuracy.

\begin{tabular}{lcccc}
\hline Model & AC & PR & RE & F1 \\
\hline LDA & 0.53822 & 0.54947 & 0.68932 & 0.60250 \\
CN-LDA & 0.54253 & 0.55386 & 0.69557 & 0.61668 \\
KNN & 0.49710 & 0.51917 & 0.72357 & 0.53035 \\
CN-KNN & 0.52877 & 0.54630 & 0.76560 & 0.56807 \\
NB & 0.53325 & 0.55452 & 0.59237 & 0.55835 \\
CN-NB & 0.53618 & 0.55374 & 0.62606 & 0.57451 \\
RF & 0.52835 & 0.54371 & 0.82920 & 0.58243 \\
CN-RF & 0.52952 & 0.54442 & 0.83224 & 0.58434 \\
SVM & 0.52342 & 0.52342 & 0.99956 & 0.68631 \\
CN-SVM & 0.52905 & 0.52905 & 1.00000 & 0.69200 \\
\hline
\end{tabular}

In conclusion, for the prediction of the EU carbon market price trend, the prediction model incorporating the network topology information can effectively improve the prediction effect of the benchmark model. Next, we compare the prediction effects of the CN-LDA, CN-KNN, $\mathrm{CN}-\mathrm{NB}, \mathrm{CN}-\mathrm{RF}$, and CN-SVM prediction models. From the perspective of prediction accuracy, the order of improvement of prediction accuracy from high to low after integrating network topology information is as follows: $\mathrm{CN}-\mathrm{KNN}>\mathrm{CN}-\mathrm{SVM}>\mathrm{CN}-\mathrm{LDA}>\mathrm{CN}-\mathrm{NB}>\mathrm{CN}-\mathrm{RF}$. The prediction accuracy of the five prediction models from high to low is CN-LDA $>\mathrm{CN}-\mathrm{NB}>\mathrm{CN}-\mathrm{RF}>\mathrm{CN}-$ $S V M>C N-K N N$. From the perspective of the F1 value of prediction accuracy, the order of improvement of the F1 value of prediction accuracy from high to low is as follows: $\mathrm{CN}-\mathrm{KNN}>\mathrm{CN}-\mathrm{NB}>\mathrm{CN}-\mathrm{LDA}>\mathrm{CN}-\mathrm{SVM}>\mathrm{CN}-$ $\mathrm{RF}$. The $\mathrm{F} 1$ value of the five prediction models from high to low order is as follows: $\mathrm{CN}-\mathrm{SVM}>\mathrm{CN}-\mathrm{LDA}>\mathrm{CN}-$ $\mathrm{RF}>\mathrm{CN}-\mathrm{NB}>\mathrm{CN}-\mathrm{KNN}$. Thus, after integrating network topology information, the prediction accuracy and F1 value of the KNN prediction model are improved most significantly.

4.3. Analysis of the Forecast Results of China's Pilot Carbon Market. The price fluctuation trend of seven pilot carbon markets in China is predicted by using five benchmark forecasting models and the forecasting model incorporating network topology information. The prediction accuracy, precision, recall, and F1 value of each prediction model are calculated, and the results are shown in Figures 5(a)-5(g). Comparison results of the average precision of carbon price prediction in seven pilot carbon markets are shown in Table 4.

As Figure 5(a) shows, for Beijing's carbon market, after integrating the network topology information, only the forecasting accuracy of CN-LDA and the forecasting accuracy, precision, and F1 score of CN-NB are improved compared with the benchmark models LDA and NB. As Figure 5(b) illustrates, for Guangdong's carbon market, after integrating the network topology information, only the forecasting precision of $\mathrm{CN}-\mathrm{KNN}$ and the forecasting accuracy and forecasting precision of CN-RF and CN-SVM are improved compared with the benchmark models KNN, RF, and SVM. Moreover, as Figure 5(c) shows, for Chongqing's carbon market, after integrating the network topology information, the prediction effects (including prediction accuracy, precision, recall, and F1 score) of the CN-KNN, $\mathrm{CN}-\mathrm{RF}$, and $\mathrm{CN}-\mathrm{SVM}$ models are significantly improved, and the forecasting precision of $\mathrm{CN}-\mathrm{NB}$ is improved compared with the benchmark model. However, the prediction effects of CN-LDA are not improved compared with the benchmark model. From Figure 5(d), for Shanghai's carbon market, after integrating the network topology information, the prediction accuracy of $\mathrm{CN}-\mathrm{RF}$ and the prediction precision of $\mathrm{CN}-\mathrm{NB}$ are improved compared with the benchmark model. However, other forecasting models fail to improve their performance. As shown in Figure 5(e), for Tianjin's carbon market, after integrating the network topology information, the prediction effects (including prediction accuracy, precision, recall, and F1 score) of the $\mathrm{CN}-\mathrm{NB}, \mathrm{CN}-\mathrm{RF}$, and CN-SVM are significantly improved compared with the benchmark model, while the CN-LDA and $\mathrm{CN}-\mathrm{KNN}$ fail to improve their performance. What needs to be pointed out here is that when the benchmark NB prediction model is used to predict the trend of carbon price fluctuation in Tianjin's carbon market, the prediction accuracy, recall, and F1 value are all very small, at 0.24492 , 0.15168 , and 0.25402 , respectively. This situation indicates that the NB model is invalid in predicting the trend of carbon price fluctuation in Tianjin's carbon market at this time. However, the CN-NB prediction model obtained by integrating the network topology information significantly improves the prediction accuracy of the NB prediction model, enabling the accuracy, recall rate, and F1 value to reach $0.56708,0.52440$, and 0.67349 , respectively. This situation also indicates that the previously invalid prediction model can become effective after integrating the network topology information. As shown in Figure 5(f), for Shenzhen's carbon market, after integrating the network topology information, the prediction effects (including prediction accuracy, precision, recall, and F1 score) of the $\mathrm{CN}-\mathrm{LDA}, \mathrm{CN}-\mathrm{KNN}$, and CN-RF are significantly improved compared with the benchmark model, while the CN-NB and $\mathrm{CN}-\mathrm{SVM}$ fail to improve their performance. As Figure 5(g) shows, for Hubei's carbon market, after integrating the network topology information, all the prediction models fail to improve their performance. In summary, for the price trend prediction of seven domestic pilot carbon markets, the prediction model integrated with network topology cannot always effectively improve the prediction accuracy of the benchmark model.

In the following, we compare and analyze the performance of different forecasting models in seven pilot carbon market price forecasts in China from the perspective of the forecasting effect. We calculate the average prediction accuracy of each prediction model and obtain the following results.

From Table 4, we find that, for Beijing's carbon market, the prediction effect of the models from the highest to the lowest is SVM (0.8301), CN-SVM (0.8300), LDA (0.8296), CN-LDA (0.8288), RF (0.8268), CN-RF (0.8257), CN-NB (0.8060), NB (0.8041), KNN (0.7995), and CN-KNN (0.7873). For Guangdong's carbon market, the prediction effect of the models from the highest to the lowest is $\mathrm{CN}$ - 


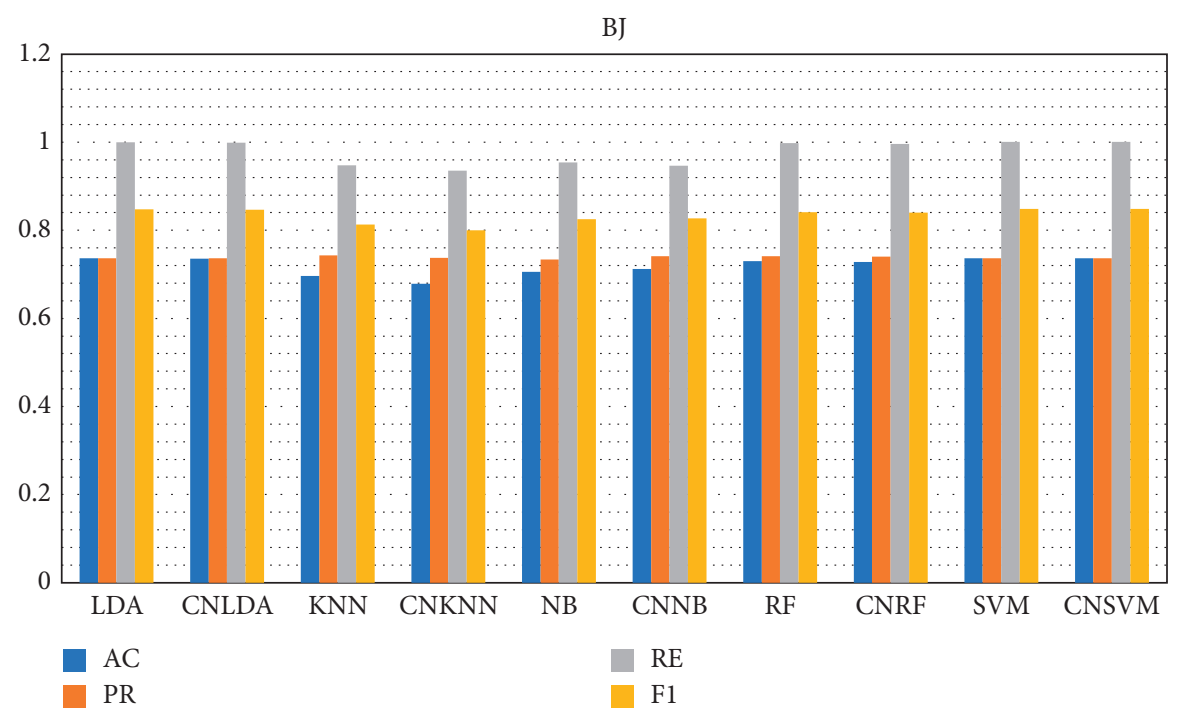

(a)

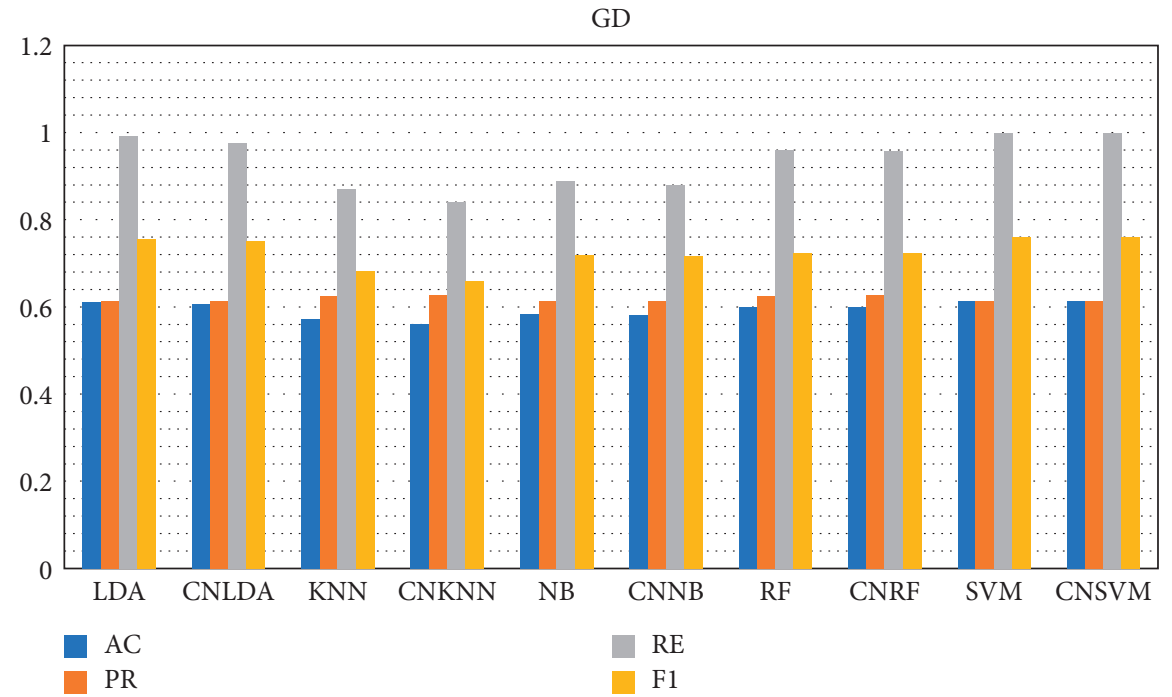

(b)

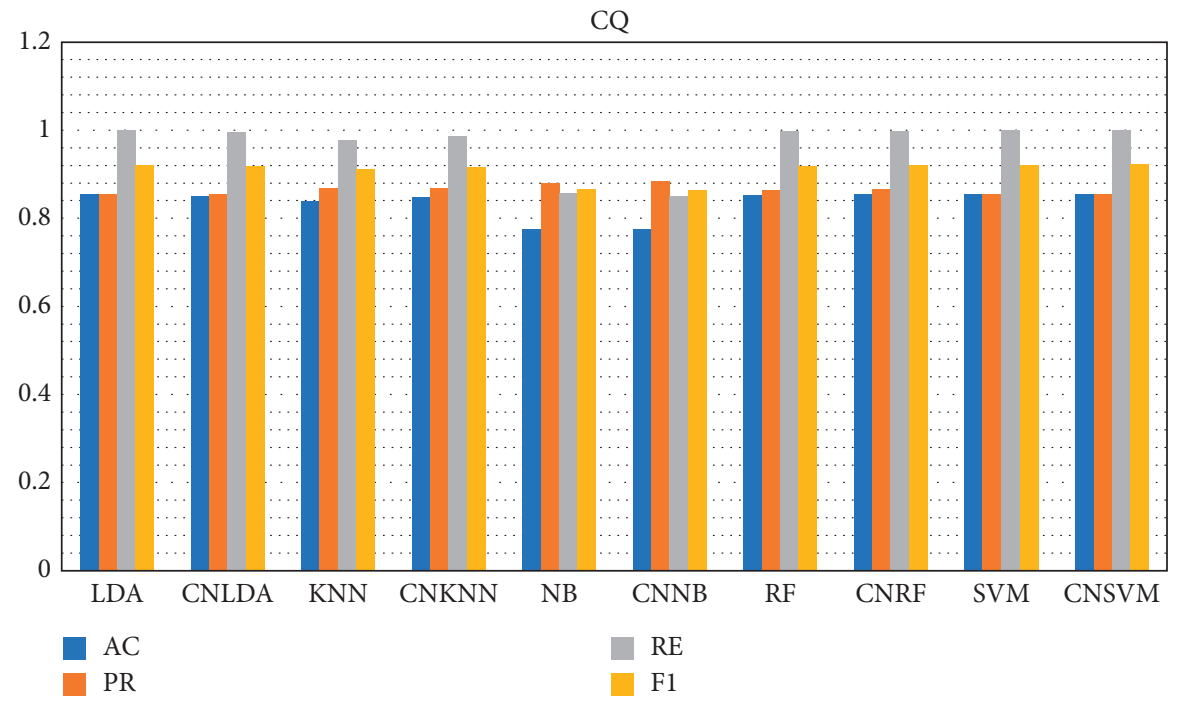

(c)

Figure 5: Continued. 


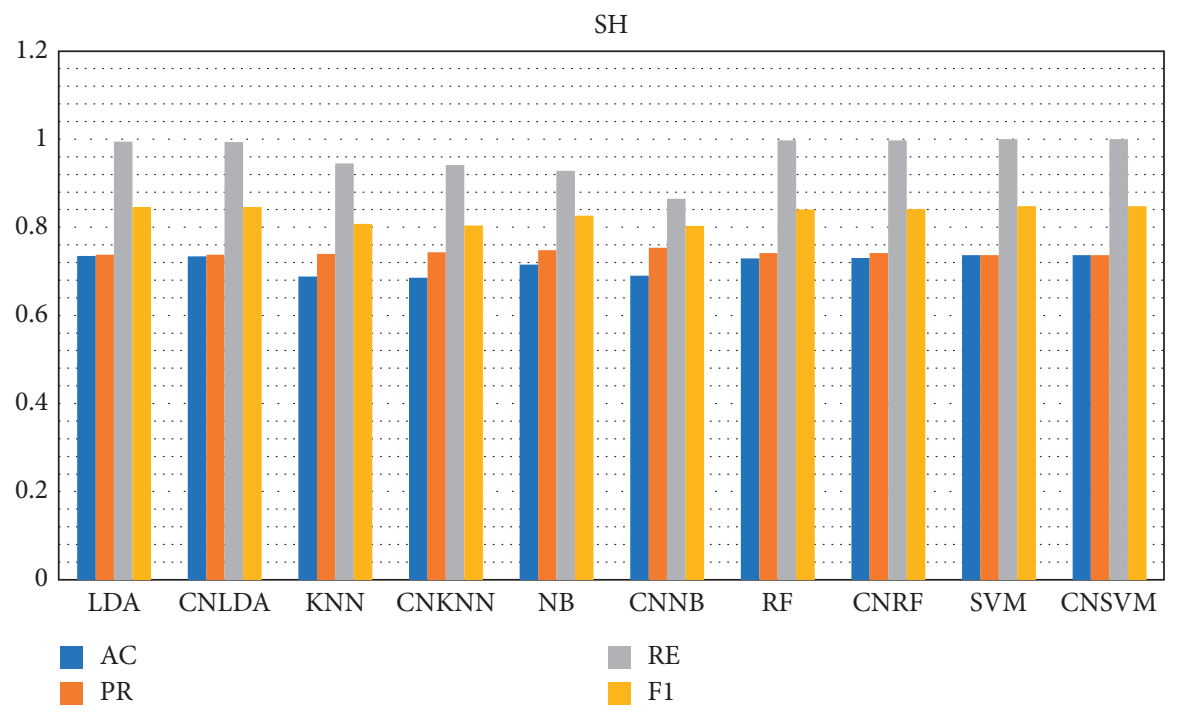

(d)

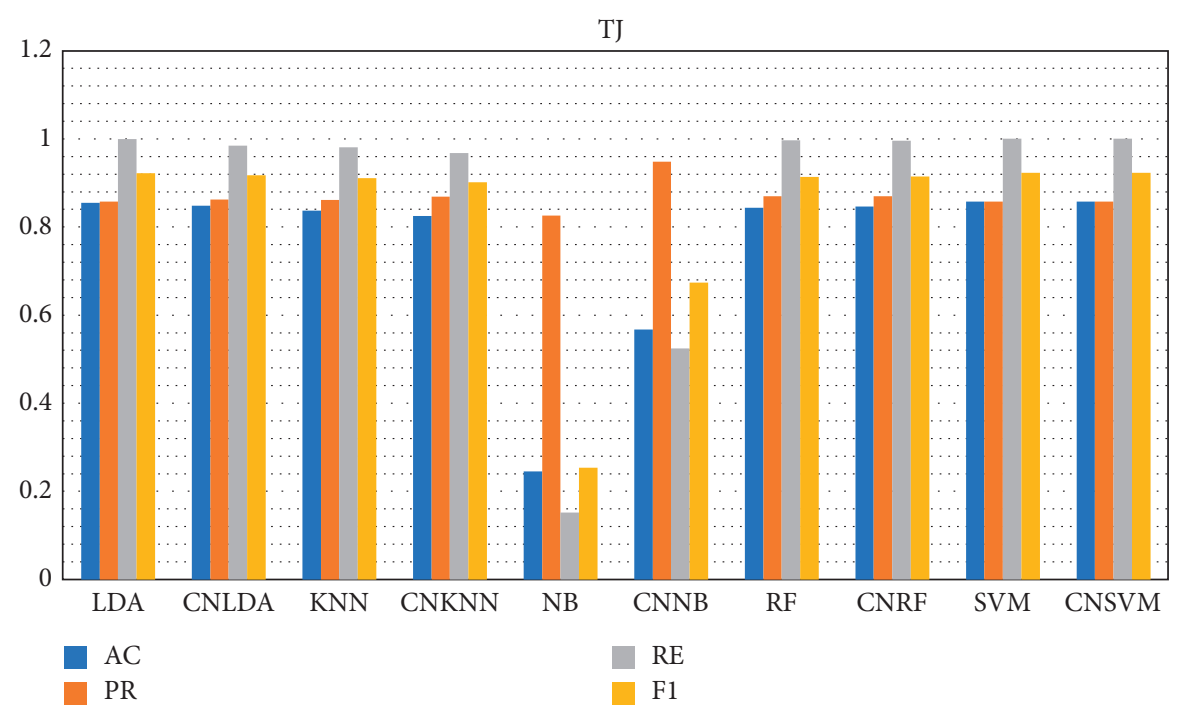

(e)

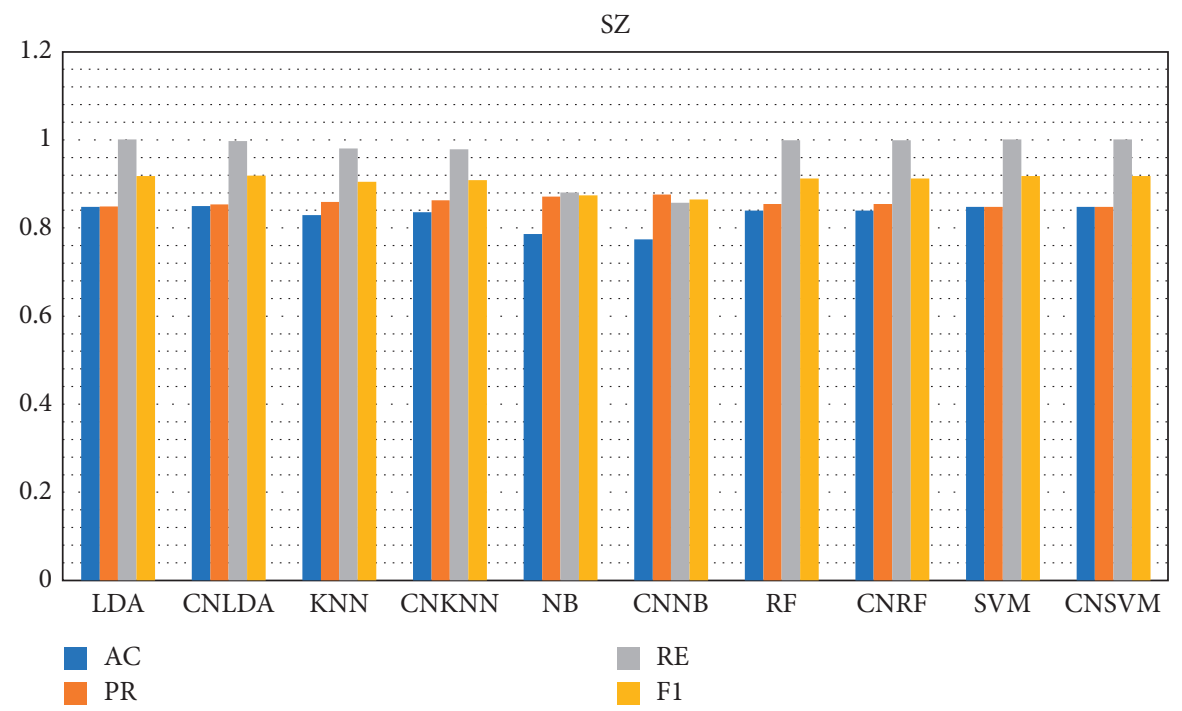

(f)

Figure 5: Continued. 


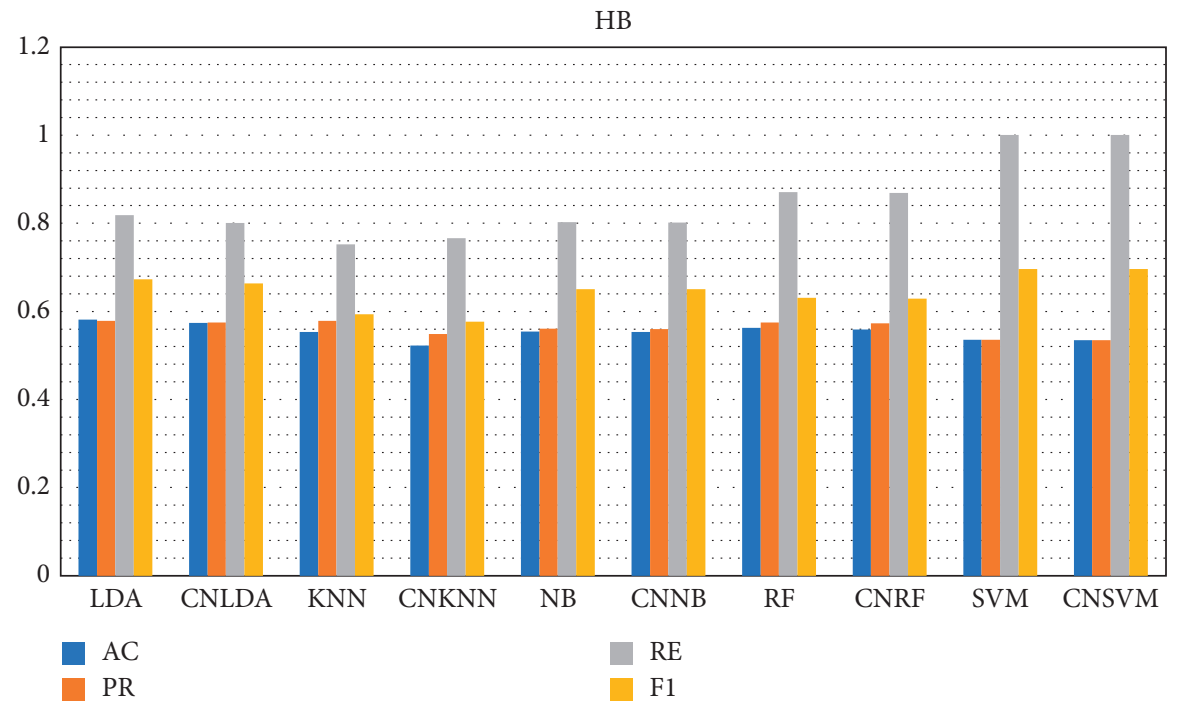

(g)

FiguRE 5: Comparison of the predicted results of different forecasting models on the fluctuation trend of carbon price in China's carbon market.

TABLE 4: The average prediction accuracy of China's pilot carbon market.

\begin{tabular}{lccccccc}
\hline & BJ & GD & CQ & SH & TJ & SZ & HB \\
\hline LDA & 0.8296 & 0.7413 & 0.9070 & 0.8286 & 0.9078 & 0.9030 & 0.6624 \\
CN- & 0.8288 & 0.7365 & 0.9043 & 0.8280 & 0.9075 & 0.9087 & 0.6586 \\
LDA & & & & & & \\
KNN & 0.7995 & 0.6877 & 0.8985 & 0.7950 & 0.8971 & 0.8925 & 0.6190 \\
CN- & 0.7873 & 0.6713 & 0.9051 & 0.7933 & 0.8901 & 0.8956 & 0.6082 \\
KNN & & & & & \\
NB & 0.8041 & 0.7016 & 0.8445 & 0.8045 & 0.3689 & 0.8519 & 0.6415 \\
CN-NB & 0.8060 & 0.6972 & 0.8433 & 0.7981 & 0.6782 & 0.8480 & 0.6410 \\
RF & 0.8268 & 0.7270 & 0.9083 & 0.8271 & 0.9055 & 0.9003 & 0.6594 \\
CN-RF & 0.8257 & 0.7275 & 0.9112 & 0.8275 & 0.9062 & 0.9002 & 0.6570 \\
SVM & 0.8301 & 0.7419 & 0.9072 & 0.8305 & 0.9092 & 0.9026 & 0.6915 \\
CN- & 0.8300 & 0.7463 & 0.9074 & 0.8305 & 0.9121 & 0.9025 & 0.6914 \\
SVM & & & & & & & \\
\hline
\end{tabular}

SVM (0.7463), SVM (0.7419), LDA (0.7413), CN-LDA (0.7365), CN-RF (0.7275), RF (0.7270), NB (0.7016), CN-NB (0.6972), KNN (0.6877), and CN-KNN (0.6713). For Chongqing's carbon market, the prediction effect of the models from the highest to the lowest is CN-RF (0.9112), RF (0.9083), CN-SVM (0.9074), SVM (0.9072), LDA (0.9070), CN-KNN (0.9051), CN-LDA (0.9043), KNN (0.8985), NB (0.8445), and CN-NB (0.8433). For Shanghai's carbon market, the prediction effect of the models from the highest to the lowest is SVM (0.8305), CN-SVM (0.8305), LDA (0.8286), CN-LDA (0.8280), CN-RF (0.8275), RF (0.8271), NB (0.8045), CN-NB (0.7981), KNN (0.7950), and CN-KNN (0.7933). For Tianjin's carbon market, the prediction effect of the models from the highest to the lowest is CN-SVM (0.9121), SVM (0.9092), LDA (0.9078), CN-LDA (0.9075), CN-RF (0.9062), RF (0.9055), KNN (0.8971), CN-KNN (0.8901), CN-NB (0.6782), and NB (0.3689). For Shenzhen's carbon market, the prediction effect of the models from the highest to the lowest is CN-LDA (0.9087), LDA (0.9030), SVM (0.9026), CN-SVM (0.9025), RF (0.9003), CN-RF (0.9002), CN-KNN (0.8956), KNN (0.8924), NB (0.8519), and CN-NB (0.8480). For Hubei's carbon market, the prediction effect of the models from the highest to the lowest is SVM (0.6915), CN-SVM (0.6914), LDA (0.6624), RF (0.6594), CN-LDA (0.6586), CN-RF (0.6570), NB (0.6415), CN-NB (0.6410), KNN (0.6190), and CN-KNN (0.6082). To sum up the statistical results, the models with the best forecasting effect for the seven pilot carbon markets in Beijing, Guangdong, Chongqing, Shanghai, Tianjin, Shenzhen, and Hubei are SVM (0.8301), CN-SVM (0.7463), CN-RF (0.9112), SVM (0.8304), CN-SVM (0.9121), CNLDA (0.9087), and SVM (0.6915), respectively. According to the model with the best carbon market prediction effect, after integrating the topology information of the carbon price network, the price trend prediction effect of the four carbon markets in Guangdong, Chongqing, Tianjin, and Shenzhen is significantly improved by $0.5931 \%, 0.3193 \%$, $0.3190 \%$, and $0.6312 \%$, respectively. For the Beijing, Shanghai, and Hubei carbon markets, the prediction accuracy of the benchmark model has almost no change after the topological structure information of the carbon price network is integrated, and the prediction accuracy varies from $0.0095 \%$ to $0.0145 \%$.

4.4. Comparative Analysis of Carbon Price Trend Prediction Results between the EU Carbon Market and Seven Domestic Pilot Carbon Markets. In the following, we make a horizontal comparison of the effect of carbon price fluctuation trend prediction in seven domestic pilot carbon markets and calculate the average accuracy, precision, recall, and F1 value of carbon price fluctuation trend prediction in the EU and seven Chinese pilot carbon markets. The results are shown in Figure 6. 


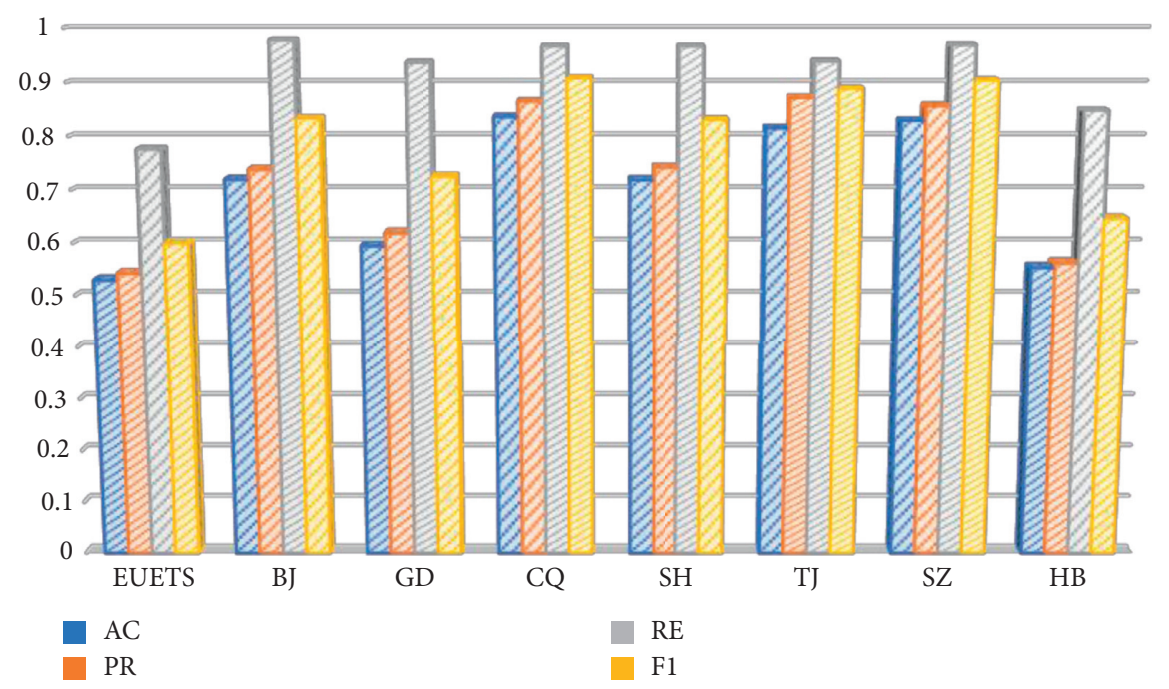

FIGURE 6: Comparison of price trend prediction effect between the EU and seven pilot carbon markets in China.

From Figure 6, we can reach the following conclusions: the prediction effect of carbon price in seven domestic pilot carbon markets is significantly higher than that in the EU ETS. The average accuracy of carbon price prediction in the EU ETS is 0.52864 , the average precision is 0.54177 , the average recall is 0.77535 , and the average F1 score is 0.59955 . The average accuracy of carbon price prediction in the seven domestic pilot carbon markets is 0.72336 , the average precision is 0.75039 , the average recall is 0.94293 , and the average $\mathrm{F} 1$ score is 0.81929 . The forecast accuracy, precision, recall, and F1 score of China's pilot carbon markets are $36.8346 \%, 38.5079 \%, 21.6134 \%$, and $36.6508 \%$ higher than those of the EU carbon market, respectively. Furthermore, when we look at the comparison of individual carbon markets, we find that the accuracy of price trend prediction of Chongqing's carbon market ranks first and is 58.0795\% higher than that of EU ETS. The accuracy of the price trend prediction of Hubei's carbon market ranks last and is $4.5587 \%$ higher than that of the EU carbon market. The precision of carbon price trend prediction in Tianjin's carbon market ranks first and is $60.9486 \%$ higher than that of EU ETS. The precision of price trend prediction in Hubei's carbon market ranks last and is $3.6619 \%$ higher than that of EU ETS. The recall of price trend prediction in Beijing's carbon market ranks first and is $26.0111 \%$ higher in accuracy than that of the EU ETS. The recall of carbon price trend prediction in Hubei's carbon market ranks last, with a 9.3414\% higher accuracy than that of the EU carbon market. The F1 score of price trend prediction of Chongqing's carbon market ranks first, with a 51.408\% higher accuracy than that of the EU ETS, and the F1 score of carbon price trend prediction of Hubei's carbon market ranks last, with a 7.6807\% higher than that of the EU ETS.

\section{Discussion}

China's pilot and EU carbon markets have huge differences in carbon price fluctuations. From the structural characteristics of the carbon price network, the EU carbon price network is an assortativity network, while the seven pilot carbon market price networks in China are all disassortativity networks. Price fluctuations in China's seven pilot carbon markets also vary widely; the carbon market price in Shanghai presents a left-skewed, flat distribution; the carbon market price in Beijing, Chongqing, Shenzhen, and Tianjin presents a right-skewed, flat distribution; and the carbon market price data in Guangdong and Hubei present a right-skewed, sharp distribution. It can be seen from the prediction results that the prediction effect of the Chinese carbon market price trend using the same model is significantly higher than that of the EU carbon market, indicating that the price fluctuation complexity of China's pilot carbon market is lower than that of the EU ETS, and the price fluctuation of China's carbon market is more regular. In fact, in this paper, we predict the future trend of carbon' price based on the historical data of each carbon market. The more effective the information that can reflect the future trend of price fluctuation in the process of historical price fluctuation, the higher the precision of carbon price trend prediction.

This paper concludes that the prediction accuracy of carbon price trend in China's pilot carbon market is significantly higher than that in the EU ETS, which indicates that the price fluctuation in China's pilot carbon market can be explained by historical carbon prices that are significantly higher than those in the EU carbon market. This conclusion is consistent with previous studies $[45,46]$. The EU carbon market is currently considered to be a relatively mature carbon market, while the carbon price signal of China's pilot carbon market has not been fully played. The main reasons for the above problems lie in the inactive trading, imperfect laws, and low marketization degree of the domestic pilot carbon market. Although the market efficiency of China's pilot carbon market shows a trend of continuous improvement, on the whole, the construction of China's carbon market is still in its infancy and will face some uncertainties. There is still a long way to go before the carbon market will explore, iterate, and mature. 
The importance of low-carbon development is being raised to an unprecedented level after China presented the world with a carbon peak and carbon neutral timetable, which will become the fundamental driving force for the development of the national carbon market. On January 1, 2021, the first implementation cycle of China's national carbon market was officially launched, involving 2,225 key emitters in the power generation sector. Making the national carbon market a more efficient and active market will be a major challenge. In the process of carbon market construction, industries including steel, cement, chemical, electrolytic aluminum, and paper should be included as soon as possible. More diversified trading parties, including asset operating institutions and financial institutions, should be added. Diversified participants will promote the formation of an active market. To achieve a reasonable price in the carbon market, the total amount should be determined first, and the carbon quota should be traded on the premise of the total amount being determined to establish regular conditions for the price formation. How do we determine and calculate the total amount of carbon? Economic growth, industrial restructuring, energy structure optimization, coordinated control of air pollutant emissions, and other factors need to be taken into account in formulating the total carbon emission quota. As for carbon price, we should give full play to the role of the market itself and reduce the unreasonable intervention of the government in the market as much as possible. However, the government should still establish rules and strict supervision, and at the same time, it should strengthen the connection between the carbon market and the financial market and strengthen the interaction with the carbon finance and capital market, which will increase the size of the carbon market and contribute to the formation of the carbon price. Through the above measures, the carbon financial market can be active, improve the efficiency of the market, and promote the carbon market as an important tool to cope with climate change and achieve carbon neutrality.

The main purpose of this paper is to discuss whether incorporating the topology of the complex transformed from the original carbon price data into the original carbon price data can help improve the prediction accuracy of the existing prediction model, rather than focusing on whether the constructed prediction model has the highest prediction accuracy. In this paper, we select six classical prediction models as benchmark models, and empirical analysis using carbon market price data shows that the prediction accuracy of the benchmark model can be significantly improved after the integration of network topology information, indicating that the idea proposed in this paper is effective. Of course, we can also choose other classical prediction models as benchmark models, such as neural networks and extreme learning machines. Although the neural network model may achieve better prediction accuracy, the neural network model itself contains many parameters such as the number of neurons, the number of hidden layers, activation function, and learning rate, and the choice of parameters has a significant impact on the prediction accuracy of the model. Therefore, in order to reduce the process of parameter tuning and verify the prediction ideas proposed in this paper more quickly, we did not select the neural network model as the benchmark model. In fact, we have built a crude oil price prediction model based on a complex network and neural network in literature [47] and verified that the hybrid model has high prediction accuracy.

In practical application, the core step of the prediction model proposed in this paper is to transform the original data into a complex network. In this paper, the transformation method we use is the visibility graph algorithm, which has the advantage of no parameters, that is, a parameterless mapping algorithm. In addition to the visibility graph algorithm, the complex network transformation algorithm of time series data also includes recurrence network, coarse-grained, and other methods, which need to determine some additional parameters. Therefore, in practical application, we recommend using the visibility graph method to transform time series data into a complex network and then use network topology to extract data information. In the following research, we will continue to study the time series prediction algorithm based on a multilayer complex network.

\section{Data Availability}

The carbon price data of EU carbon market (EUETS) and seven pilot carbon markets of China from June 19, 2014, to October 9, 2020, that is, Beijing (BJ), Guangdong (GD), Chongqing (CQ), Shanghai (SH), Tianjin (TJ), Shenzhen (SZ), and Hubei (HB), were selected as sample data.

\section{Conflicts of Interest}

The authors declare that they have no conflicts of interest.

\section{Acknowledgments}

The research was supported by the following foundations: National Key R\&D Program of China under Grant No. 2020YFA0608602, Qing Lan Project of Jiangsu Province (2021), Six Talent Peaks Project in Jiangsu Province (JY055), and China Postdoctoral Science Foundation Funded Project (2021M691312).

\section{References}

[1] United Nations Environment Programme (Unep), Emissions Gap Report 2020, United Nations Environment Programme and UNEP DTU Partnership, Denmark, Europe, 2020.

[2] Y. M. Wei, R. Han, C. Wang et al., "Self-preservation strategy for approaching global warming targets in the post-Paris Agreement era," Nature Communications, vol. 11, no. 1, 2020.

[3] G. Koop and L. Tole, "Forecasting the European carbon market," Journal of the Royal Statistical Society: Series A, vol. 176, no. 3, pp. 723-741, 2013.

[4] H. N. Guðbrandsdóttir and H. Ó Haraldsson, "Predicting the price of EU ETS carbon credits," Systems Engineering Procedia, vol. 1, pp. 481-489, 2011.

[5] A. Abdi and S. Taghipour, "Forecasting carbon price in the Western Climate Initiative market using Bayesian networks," Carbon Management, vol. 10, no. 3, pp. 255-268, 2019. 
[6] Y. Song, T. Liu, B. Ye, Y. Zhu, Y. Li, and X. Song, "Improving the liquidity of China's carbon market: insight from the effect of carbon price transmission under the policy release," Journal of Cleaner Production, vol. 239, Article ID 118049, 2019.

[7] A. Lanza, M. Manera, and M. Giovannini, "Modeling and forecasting cointegrated relationships among heavy oil and product prices," Energy Economics, vol. 27, no. 6, pp. 831-848, 2005.

[8] A. Murat and E. Tokat, "Forecasting oil price movements with crack spread futures," Energy Economics, vol. 31, no. 1, pp. 85-90, 2009.

[9] C. Baumeister and L. Kilian, "Real-time forecasts of the real price of oil," Journal of Business \& Economic Statistics, vol. 30, no. 2, pp. 326-336, 2012.

[10] M. Segnon, T. Lux, and R. Gupta, "Modeling and forecasting the volatility of carbon dioxide emission allowance prices: a review and comparison of modern volatility models," Renewable and Sustainable Energy Reviews, vol. 69, pp. 692-704, 2017.

[11] Y. Xiang and X. H. Zhuang, "Application of ARIMA model in short-term prediction of international crude oil price," Advanced Materials Research, vol. 798-799, pp. 979-982, 2013.

[12] Y. Fan, Y. J. Zhang, H. T. Tsai, and Y. M. Wei, "Estimating 'Value at Risk' of crude oil price and its spillover effect using the GED-GARCH approach," Energy Economics, vol. 30, no. 6, pp. 3156-3171, 2008.

[13] H. Mohammadi and L. Su, "International evidence on crude oil price dynamics: applications of ARIMA-GARCH models," Energy Economics, vol. 32, no. 5, pp. 1001-1008, 2010.

[14] G. S. Atsalakis, "Using computational intelligence to forecast carbon prices," Applied Soft Computing, vol. 43, pp. 107-116, 2016.

[15] M. Yahşi, E. Çanakoğlu, and S. Ağralı, "Carbon price forecasting models based on big data analytics," Carbon Management, vol. 10, no. 2, pp. 175-187, 2019.

[16] W. Xie, L. Yu, S. Xu, and S. Wang, "A new method for crude oil price forecasting based on support vector machines," in Proceedings of the Computational Science - ICCS 2006, pp. 444-451, Reading, United Kingdom, May 2006.

[17] B. Zhu, D. Han, P. Wang, Z. Wu, T. Zhang, and Y. M. Wei, "Forecasting carbon price using empirical mode decomposition and evolutionary least squares support vector regression," Applied Energy, vol. 191, pp. 521-530, 2017.

[18] H. Lu, X. Ma, K. Huang, and M. Azimi, "Carbon trading volume and price forecasting in China using multiple machine learning models," Journal of Cleaner Production, vol. 249, Article ID 119386, 2020.

[19] B. Zhu and Y. Wei, "Price forecast of international carbon market based on GMDH-PSO-LSSVM," Systems Engineering Theory and Practice, vol. 31, no. 12, pp. 2264-2271, 2011.

[20] Y. Gao and J. Li, "Price forecast of international carbon financial market based on EMD-PSO-SVM error correction model," Population, Resources and Environment in China, vol. 6, pp. 163-170, 2014.

[21] B. Zhu and Y. Wei, "Carbon price forecasting with a novel hybrid ARIMA and least squares support vector machines methodology," Omega, vol. 41, no. 3, pp. 517-524, 2013.

[22] L. Zhang, J. Zhang, T. Xiong, and C. Su, "Interval forecasting of carbon futures prices using a novel hybrid approach with exogenous variables," Discrete Dynamics in Nature and Society, vol. 2017, Article ID 5730295, 12 pages, 2017.

[23] X. Shi and B. Zhu, "Carbon market price prediction based on phase space reconstruction and least square support vector regression model parameter synchronization optimization,"
Systems Science and Mathematics, vol. 37, no. 2, pp. 562-572, 2017.

[24] B. Zhu, S. Ye, P. Wang, K. He, T. Zhang, and Y. M. Wei, “A novel multiscale nonlinear ensemble leaning paradigm for carbon price forecasting," Energy Economics, vol. 70, pp. 143-157, 2018.

[25] J. Zhu, P. Wu, H. Chen, J. Liu, and L. Zhou, "Carbon price forecasting with variational mode decomposition and optimal combined model," Physica A: Statistical Mechanics and Its Applications, vol. 519, pp. 140-158, 2019.

[26] W. Sun and C. Huang, "A carbon price prediction model based on secondary decomposition algorithm and optimized back propagation neural network," Journal of Cleaner Production, vol. 243, Article ID 118671, 2020.

[27] Y. Huang, J. Hu, H. Liu, and S. Liu, "Research on price forecasting method of China's carbon trading market based on PSO-RBF algorithm," Systems Science \& Control Engineering, vol. 7, no. 2, pp. 40-47, 2019.

[28] J. Zhou, X. Huo, X. Xu, and Y. Li, "Forecasting the carbon price using extreme-point symmetric mode decomposition and extreme learning machine optimized by the Grey Wolf optimizer algorithm," Energies, vol. 12, no. 5, 2019.

[29] H. Liu and L. Shen, "Forecasting carbon price using empirical wavelet transform and gated recurrent unit neural network," Carbon Management, vol. 11, no. 2, pp. 1-13, 2019.

[30] L. Lacasa, B. Luque, F. Ballesteros, J. Luque, and J. C. Nuño, "From time series to complex networks: the visibility graph," Proceedings of the National Academy of Sciences, vol. 105, no. 13 , pp. 4972-4975, 2008.

[31] J. Zhang and M. Small, "Complex network from pseudoperiodic time series: topology versus dynamics," Physical Review Letters, vol. 96, no. 23, Article ID 238701, 2006.

[32] X. Xu, J. Zhang, and M. Small, "Superfamily phenomena and motifs of networks induced from time series," Proceedings of the National Academy of Sciences, vol. 105, no. 50, Article ID 19601, 2008.

[33] M. Wang and L. Tian, "From time series to complex networks: the phase space coarse graining," Physica A: Statistical Mechanics and Its Applications, vol. 461, pp. 456-468, 2016a.

[34] M. Wang, Y. Chen, L. Tian, S. Jiang, Z. Tian, and R. Du, "Fluctuation behavior analysis of international crude oil and gasoline price based on complex network perspective," Applied Energy, vol. 175, pp. 109-127, 2016b.

[35] H. An, X. Gao, W. Fang, X. Huang, and Y. Ding, "The role of fluctuating modes of autocorrelation in crude oil prices," Physica A: Statistical Mechanics and Its Applications, vol. 393, pp. 382-390, 2014.

[36] G. Dong, T. Qing, R. Du et al., "Complex network approach for the structural optimization of global crude oil trade system," Journal of Cleaner Production, vol. 251, Article ID 119366, 2020.

[37] J. B. Geng, Y. J. Du, Q. Ji, and D. Zhang, "Modeling return and volatility spillover networks of global new energy companies," Renewable and Sustainable Energy Reviews, vol. 135, Article ID 110214, 2021.

[38] B. Luque, L. Lacasa, and F. J. Ballesteros, "Horizontal visibility graphs: exact results for random time series," Physical Review, vol. 80, Article ID 046103, 2010.

[39] D. J. Watts and S. H. Strogatz, "Collective dynamics of 'smallworld' networks," Nature, vol. 393, no. 6684, pp. 440-442, 1998.

[40] M. E. J. Newman, “Assortative mixing in networks," Physical Review Letters, vol. 89, no. 20, Article ID 208701, 2002. 
[41] F. Wang, Q. Wang, and F. Nie, "Unsupervised linear discriminant analysis for jointly clustering and subspace learning," IEEE Transactions on Knowledge and Data Engineering, vol. 33, no. 3, pp. 1276-1290, 2021.

[42] I. Vidal and M. D. Castro, "A Bayesian analysis of the matching problem," Journal of Statistical Planning and Inference, vol. 212, pp. 194-200, 2020.

[43] Z. Pan, Y. Wang, and Y. Pan, "A new locally adaptive k-nearest neighbor algorithm based on discrimination class," Knowledge-Based Systems, vol. 204, Article ID 106185, 2020.

[44] Q. Gu, Y. Chang, X. Li, and Z. Chang, "A novel F-SVM based on FOA for improving SVM performance," Expert Systems with Applications, vol. 165, Article ID 113713, 2020.

[45] J. Fan and N. Todorova, "Dynamics of China's carbon prices in the pilot trading phase," Applied Energy, vol. 208, no. 12, pp. 1452-1467, 2017.

[46] X. Tan, K. Sirichand, A. Vivian, and X. Wang, "How connected is the carbon market to energy and financial markets? A systematic analysis of spillovers and dynamics," Energy Economics, vol. 90, Article ID 104870, 2020.

[47] M. Wang, L. Zhao, R. Du et al., "A novel hybrid method of forecasting crude oil prices using complex network science and artificial intelligence algorithms," Applied Energy, vol. 220, pp. 480-495, 2018. 